\title{
The effect of sudden source buoyancy flux increases on turbulent plumes
}

\author{
M. M. SCASE $\operatorname{SC}^{1}$, A. J. ASPDEN ${ }^{2}$ AND C. P. CAULFIELD $D^{3,4}$ \\ ${ }^{1}$ Division of Process and Environmental Engineering, Faculty of Engineering, \\ University of Nottingham, Nottingham NG7 2RD, UK \\ ${ }^{2}$ Lawrence Berkeley National Laboratory, 1 Cyclotron Road, Berkeley, CA 94720, USA \\ ${ }^{3}$ BP Institute, University of Cambridge, Madingley Road, Cambridge CB3 OEZ, UK \\ ${ }^{4}$ Department of Applied Mathematics and Theoretical Physics, University of Cambridge, \\ Centre for Mathematical Sciences, Wilberforce Road, Cambridge CB3 OWA, UK
}

(Received 18 August 2008; revised 23 March 2009; accepted 25 March 2009)

Building upon the recent experimentally verified modelling of turbulent plumes which are subject to decreases in their source strength (Scase et al., J. Fluid Mech., vol. 563, $2006 b$, p. 443), we consider the complementary case where the plume's source strength is increased. We consider the effect of increasing the source strength of an established plume and we also compare time-dependent plume model predictions for the behaviour of a starting plume to those of Turner (J. Fluid Mech., vol. 13, 1962, p. 356).

Unlike the decreasing source strength problems considered previously, the relevant solution to the time-dependent plume equations is not a simple similarity solution. However, scaling laws are demonstrated which are shown to be applicable across a large number of orders of magnitude of source strength increase. It is shown that an established plume that is subjected to an increase in its source strength supports a self-similar 'pulse' structure propagating upwards. For a point source plume, in pure plume balance, subjected to an increase in the source buoyancy flux $F_{0}$, the rise height of this pulse in terms of time $t$ scales as $t^{3 / 4}$ while the vertical extent of the pulse scales as $t^{1 / 4}$. The volume of the pulse is shown to scale as $t^{9 / 4}$. For plumes in pure plume balance that emanate from a distributed source it is shown that the same scaling laws apply far from the source, demonstrating an analogous convergence to pure plume balance as that which is well known in steady plumes. These scaling law predictions are compared to implicit large eddy simulations of the buoyancy increase problem and are shown to be in good agreement.

We also compare the predictions of the time-dependent model to a starting plume in the limit where the source buoyancy flux is discontinuously increased from zero. The conventional model for a starting plume is well approximated by a rising turbulent, entraining, buoyant vortex ring which is fed from below by a 'steady' plume. However, the time-dependent plume equations have been defined for top-hat profiles assuming only horizontal entrainment. Therefore, this system cannot model either the internal dynamics of the starting plume's head or the extra entrainment of ambient fluid into the head due to the turbulent boundary of the vortex ring-like cap. We show that the lack of entrainment of ambient fluid through the head of the starting plume means that the time-dependent plume equations overestimate the rise height of a starting 
plume with time. However, by modifying the entrainment coefficient appropriately, we see that realistic predictions consistent with experiment can be attained.

\section{Introduction}

In the field of turbulent plume and jet dynamics the model of Morton, Taylor \& Turner (1956) has dominated for over 50 years. Motivated by meteorological observations, in particular plume-puff transition, a time-dependent turbulent plume and jet model has recently been developed and presented (Scase et al. 2006b). This time-dependent model is based on the Morton et al. (1956) model and has been successfully compared to laboratory turbulent plumes that experience a rapid decrease in their source strength (Scase, Caulfield \& Dalziel 2008) (usually, but not exclusively, we take 'source strength' to refer to the source buoyancy flux). However, the timedependent system of equations is not restricted to modelling source buoyancy flux reductions, and in the present paper we look at the effects of buoyancy increases on turbulent plumes.

\subsection{Review of time-dependent turbulent plume models}

The time-dependent model is a generalization of the well-established 'steady' plume model of Morton et al. (1956). To date, research based on this time-dependent model has focused primarily on the effects of reducing the 'source strength' of the plume, to investigate the so-called 'plume-puff' transition. It has been observed, in areas such as the deserts of the US, that a plume, once established, persists and remains in a given location for long periods of time (see Scorer 1954). However, it has also been observed in countries such as the UK, that although plumes can become established, they often only persist for a short time, before being observed to 'pinch-off' into an isolated thermal. One possible explanation is as follows. Radiation from the sun passes through our atmosphere, which is largely transparent to the sun's rays. The radiation is absorbed by the ground which then re-emits the energy at a different wavelength, warming the surrounding air. Variations in the ground's albedo (due to variation in colour as a result of vegetation or highway infrastructure for example) can cause 'hot-spots' to form which can, in turn, trigger the formation of a warm buoyant patch of fluid just above the ground. As this buoyant patch warms and convects vertically away it removes energy from the ground. If the ground is conductive enough, more energy can be supplied to the surrounding air beneath the buoyant patch and a plume can become established. However, if the ground is not conductive enough the cool ground cannot supply any buoyancy to the surrounding air and the buoyant patch rises as an isolated thermal. For a full discussion, with diagrams, see Hunt et al. (2003).

In Scase et al. (2006b) the authors conjectured that if an established plume, obeying the steady Morton et al. (1956) model, was subjected to a sudden reduction in source buoyancy flux, a narrow transient region would form in the plume. The Morton et al. (1956) model predicts a steady plume will have radius $b(z)=6 \alpha z / 5$, where $\alpha$ is the 'entrainment constant' relating the velocity of ambient fluid entrained horizontally into the plume, at a given height, to the mean local vertical velocity of the plume. The narrow transient region in the time-dependent case was predicted to have a radius given by $b(z)=2 \alpha z / 3$. This prediction has recently been observed and supported experimentally (Scase et al. 2008). The time-dependent model was shown to be a 
good predictor of the plume radius, velocity and tracer concentration throughout the height of the plume.

The model presented in Scase et al. (2006b) considered not only point-source Boussinesq plumes propagating through homogeneous ambient fluids, but also plumes with distributed sources (in some circumstances referred to as 'lazy plumes'), nonBoussinesq plumes and stratified ambient fluids. In Scase, Caulfield \& Dalziel (2006a) it was predicted that a Boussinesq plume rising through a uniformly stratified fluid of density $\rho_{\infty}$, with buoyancy frequency $N=\left\{-g / \rho_{\infty} \mathrm{d} \rho_{\infty} / \mathrm{d} z\right\}^{1 / 2}$, where $g$ is the acceleration due to gravity, would stall and collapse at a time $t=\pi / N$ after its source strength is suddenly reduced. This prediction has had some initial experimental support (Scase et al. 2006c), but is yet to be exhaustively tested. Given a value of $N \sim 10^{-2} \mathrm{~s}^{-1}$ in the troposphere, this predicts a collapse time, or 'rain-out' time from a volcanic eruption of order $5 \mathrm{~min}$ after the eruption has ceased.

The key prediction of the theory is that while a plume remains turbulent, and the entrainment assumption can be considered to hold, the plume cannot be separated into puffs by a sharp reduction in source strength - a conclusion supported experimentally in Scase et al. (2008). However, a plume in a stratified ambient can be made to stall and collapse by such a reduction in source strength. This suggests that the plume-puff transition is associated with an as-yet unmodelled physical process above and beyond a mere reduction in source strength. To understand plume-puff transition successfully it may be necessary to set a clear threshold concentration for what may be considered plume fluid or ambient fluid. In its existing form, the time-dependent plume model regards any fluid of differing concentration or density to the ambient fluid 'at infinity' as 'plume fluid'. It is to be expected therefore that a model that explicitly assumes the plume to be entraining at all times predicts that it is impossible to form a region containing absolutely no plume fluid, i.e. it is impossible to separate the plume into puffs. Further investigations are required. Possible areas of investigation include the effects of a non-constant entrainment constant (see e.g. Kaminski et al. 2005), the influence of a non-passive, i.e. strongly advecting or convecting, ambient fluid and the role of vertical velocity in the entraining eddies at the plume edge, which are not modelled.

On the other hand, it is a completely open question as to what happens when the source strength of an established plume is increased. We address this question in the present paper. We consider both the effect of increasing the source strength of an established plume, and we also examine how successful the time-dependent model is in capturing the bulk behaviour of a starting-plume (see e.g. Turner 1962; Middleton 1975), which is the natural limit of a very large increase in source strength. The effect of buoyancy increases on established plumes and the understanding of starting plumes has a large number of important applications. These include, but are not limited to, turbulent mixing and dispersion in the atmosphere with application to industrial accidents such as the fallout ejected by the nuclear accidents at Chernobyl, Three Mile Island and Mihama. Further applications include the explosive release of toxins (e.g. the Buncefield fuel depot disaster 2005 or sarin gas attacks on the Tokyo Metro 1995), volcanic eruptions, large-scale forest fires and cloud formation.

\subsection{The time-dependent plume model}

Dimensional quantities will be denoted by a subscript $\star$ throughout unless otherwise stated, all other quantities are non-dimensional. The time-dependent plume model was derived in Scase et al. (2006b) from the vertical Euler equation using two key assumptions. Firstly it was assumed that the plume was slender, i.e. the ratio of plume width at a given height, $b_{\star}\left(z_{\star}\right)$, to $z_{\star}$ was much smaller than 1 . Typically this ratio is of 
order 0.1. This assumption allows the vertical pressure gradient to be ignored, and so the fluid pressure may be ignored throughout (as the horizontal Euler equation is not exploited). The second key assumption is the well-known 'entrainment assumption' that relates the horizontal velocity of the ambient fluid at the plume's edge $u_{e \star}$ to the plume's vertical velocity at the same height $w_{\star}$. The non-Boussinesq form of the entrainment assumption, given by Ricou \& Spalding (1961), was employed, specifically

$$
u_{e \star}=-\alpha^{(P)}\left(\frac{\rho_{\star}}{\rho_{\infty_{\star}}}\right)^{1 / 2} w_{\star} .
$$

Here we use a superscript $\mathrm{P}$, denoting a plume quantity, to avoid confusion with entrainment into thermals in later discussions in $\S 4$. The fluid density within the plume is $\rho_{\star}\left(z_{\star}, t_{\star}\right)$ and the ambient fluid density is taken to be the constant $\rho_{\text {co* }}$. Taking the Boussinesq limit (1.1) becomes the usual Morton et al. (1956) entrainment assumption.

Top-hat mass, momentum and buoyancy fluxes are defined, respectively, as

$$
\begin{gathered}
Q_{\star}=\int_{0}^{2 \pi} \int_{0}^{b_{\star}} \rho_{\star} w_{\star} r_{\star} \mathrm{d} r_{\star} \mathrm{d} \theta, \quad M_{\star}=\int_{0}^{2 \pi} \int_{0}^{b_{\star}} \rho_{\star} w_{\star}^{2} r_{\star} \mathrm{d} r_{\star} \mathrm{d} \theta, \\
F_{\star}=\int_{0}^{2 \pi} \int_{0}^{b_{\star}} g_{\star}\left(\rho_{\infty{ }^{*}}-\rho_{\star}\right) w_{\star} r_{\star} \mathrm{d} r_{\star} \mathrm{d} \theta,
\end{gathered}
$$

where $\left(r_{\star}, \theta, z_{\star}\right)$ are cylindrical polar coordinates with $z_{\star}$ aligned in the direction in which gravity is acting. The governing equations of motion are then

$$
\begin{gathered}
\frac{\partial}{\partial t_{\star}}\left(\frac{Q_{\star}^{2}}{M_{\star}}\right)+\frac{\partial Q_{\star}}{\partial z_{\star}}=2 \alpha^{(P)}\left(\pi \rho_{\infty \star \star}\right)^{1 / 2} M_{\star}^{1 / 2}, \\
\frac{\partial Q_{\star}}{\partial t_{\star}}+\frac{\partial M_{\star}}{\partial z_{\star}}=\frac{Q_{\star} F_{\star}}{M_{\star}}, \\
\frac{\partial}{\partial t_{\star}}\left(\frac{Q_{\star} F_{\star}}{M_{\star}}\right)+\frac{\partial F_{\star}}{\partial z_{\star}}=0,
\end{gathered}
$$

since the ambient fluid is homogeneous. The Boussinesq plume radius, vertical velocity and reduced gravity are given, respectively, by

$$
b_{\star}=\frac{Q_{\star}}{\sqrt{\pi \rho_{0 \triangleright \star} M_{\star}}}, \quad w_{\star}=\frac{M_{\star}}{Q_{\star}}, \quad g_{\star}^{\prime}=g_{\star} \frac{\rho_{\infty \star \star}-\rho_{\star}}{\rho_{\infty}}=\frac{F_{\star}}{Q_{\star}} .
$$

Governing equations (1.3) may be non-dimensionalized by a reference buoyancy flux $F_{00 \star}$ and a length scale $z_{\ell \star}$. We set

$$
\begin{gathered}
Q_{\star}=\left(4 \alpha^{(P)} \pi \rho_{\infty{ }_{\infty}}\right)^{2 / 3} F_{00 \star}^{1 / 3} z_{\ell \star}^{5 / 3} Q, \quad M_{\star}=\left(4 \alpha^{(P)} \pi \rho_{\infty \star \star}\right)^{1 / 3} F_{00 \star}^{2 / 3} z_{\ell \star}^{4 / 3} M, \quad F_{\star}=F_{00 \star} F, \\
z_{\star}=z_{\ell \star} z, \quad t_{\star}=\left(4 \alpha^{(P)} \pi \rho_{\infty{ }_{\infty}}\right)^{1 / 3} F_{00 \star}^{-1 / 3} z_{\ell \star}^{4 / 3} t .
\end{gathered}
$$

The non-dimensional governing equations have the same form as (1.3) with the factor $2 \alpha^{(P)}\left(\pi \rho_{\infty \circ *}\right)^{1 / 2}$ in $(1.3 a)$ replaced by 1 . (For further details of the nondimensionalization see Scase et al. 2008.)

As we shall see, the assumption that the plume is slender is not strictly valid at all times. However, given the success of the steady Morton et al. (1956) plume model in stratified ambient background fluids, and the apparent success of the time-dependent 
model, also in stratified ambient fluids, where the plume cannot be considered slender, we proceed.

\subsection{Summary of paper}

The layout of the paper is as follows: in $\S 2$ we consider the solutions to the time-dependent plume equations when a step buoyancy increase is applied to an established plume and we examine the scaling laws that govern the evolution of the adjustment to the new source conditions. This can be regarded as analogous to the buoyancy decreases considered in Scase et al. (2006b). We consider solutions to both point source plumes and plumes with distributed sources. In $\S 3$ we report on high-resolution implicit large eddy simulations (ILES) of step buoyancy increases applied to an established plume and compare the results to the model predictions of $\S 2$. Then in $\S 4$ we consider the natural limiting case of the time-dependent plume equations (i.e. as the step buoyancy increase at the source becomes arbitrarily large) to the behaviour of starting plumes, in particular in relation to the model of Turner (1962). In $\S 5$ we draw our conclusions.

\section{An increase in the source buoyancy flux of a turbulent plume}

\subsection{Introduction}

In Scase et al. (2006b) the case of an established pure plume subjected to a rapid reduction in its source buoyancy flux by a factor of 20 was considered. The plume source conditions remained 'pure' for all time in the sense that the plume source laziness $\Gamma_{0}$ defined by

$$
\Gamma_{0}=\frac{5}{8 \alpha^{(P)}\left(\pi \rho_{\infty \star}\right)^{1 / 2}} \frac{Q_{0 \star}^{2} F_{0 \star}}{M_{0 \star}^{5 / 2}},
$$

(or equivalently $\left.\Gamma_{0}=(5 / 4) Q_{0}^{2} F_{0} / M_{0}^{5 / 2}\right)$ was equal to unity throughout, where subscript 0 indicates evaluation at the source, $z=0$. As described in the Appendix of Scase et $a l$. (2006b), the numerical solution to the time-dependent plume model was found by considering perturbations to a steady plume. By reducing the source buoyancy flux by a factor of 20 , the perturbation to the source momentum flux must be reduced by a factor $20^{2 / 3}$ and the perturbation to the source mass flux must be reduced by a factor $20^{1 / 3}$, in order to maintain pure plume balance at the source, $\Gamma_{0}=1$.

In the present paper we examine a number of different strength buoyancy flux increases to an established steady plume. In each case we consider an initially 'weak' plume which has its source buoyancy flux increased to a non-dimensional source buoyancy flux 1. At $t=0$ the source buoyancy flux is increased from $F(z=0, t=-0)=F_{0}$ to $F(z=0, t=+0)=F_{1}=1$, where subscript 1 indicates evaluation at the source after the buoyancy flux increases. We primarily consider seven separate solutions for initial values of $F_{0}=10^{-7}$ increasing by factors of $10 \mathrm{up}$ to $F_{0}=10^{-1}$, and examine the evolution of the properties of the plume. Independence of the solution to numerical resolution is demonstrated in appendix B.

The non-dimensional forms of the governing equations are solved numerically for $0 \leqslant z \leqslant 7$ and $0 \leqslant t \leqslant 10$ with $z$ discretized using greater than $n_{z}=5 \times 10^{3}$ cells and $t$ discretized using $n_{t}=2.5 \times 10^{5}$ cells. Details are given in table 1 . Figure 1 shows typical plots of the evolution of the three primary dependent variables, $Q, M$ and $F$ against height $z$. The thick solid line corresponds to the solution at a time $t=0.50$ after the source buoyancy flux has been increased from $F_{0}=10^{-3}$ to $F_{1}=1$. Earlier solutions for $t=0.13,0.25$ and 0.38 are shown as dashed lines. The mass flux and 


$\begin{array}{cccccccl}n_{z} & n_{t} & F_{0} & F_{1} & \lambda^{\prime} & \lambda & k & \text { Comment } \\ - & - & 1 & 1 & \sqrt{20 / 9} & 1 & 3 / 4 & \text { Morton } \text { et al. }(1956) \\ 10^{4} & 2.5 \times 10^{5} & 10^{0} & 1 & 1.564 & 1.049 & 0.730 & \text { Model } \\ 10^{4} & 2.5 \times 10^{5} & 10^{-1} & 1 & 1.392 & 0.934 & 0.755 & \text { Model } \\ 10^{4} & 2.5 \times 10^{5} & 10^{-2} & 1 & 1.254 & 0.841 & 0.755 & \text { Model } \\ 10^{4} & 2.5 \times 10^{5} & 10^{-3} & 1 & 1.193 & 0.801 & 0.756 & \text { Model } \\ 10^{4} & 2.5 \times 10^{5} & 10^{-4} & 1 & 1.165 & 0.782 & 0.758 & \text { Model } \\ 5 \times 10^{3} & 2.5 \times 10^{5} & 10^{-5} & 1 & 1.150 & 0.771 & 0.758 & \text { Model } \\ 5 \times 10^{3} & 2.5 \times 10^{5} & 10^{-6} & 1 & 1.139 & 0.764 & 0.758 & \text { Model } \\ 5 \times 10^{3} & 2.5 \times 10^{5} & 10^{-7} & 1 & 1.131 & 0.759 & 0.760 & \text { Model } \\ - & - & 10^{-\infty} & 1 & 1.123 & 0.753 & - & \text { Extrapolated } \\ - & - & 0 & 1 & 0.91 & 0.61 & 3 / 4 & \text { Turner }(1962)\end{array}$

TABLE 1. Tabulated results from numerical solutions to our model. Using the errors in the power $k$, compared to the steady Morton et al. (1956) solution, the errors at this high resolution are of order $2 \%$. The resolution in the spatial and temporal directions is given by $n_{z}$ and $n_{t}$ respectively.

(a)

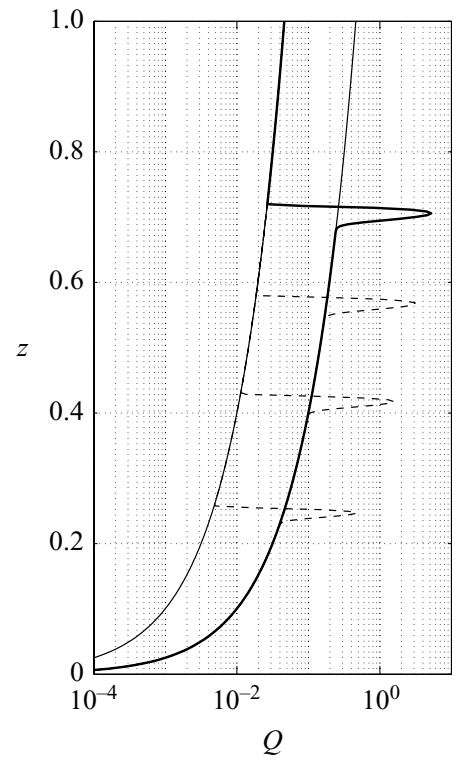

(b)

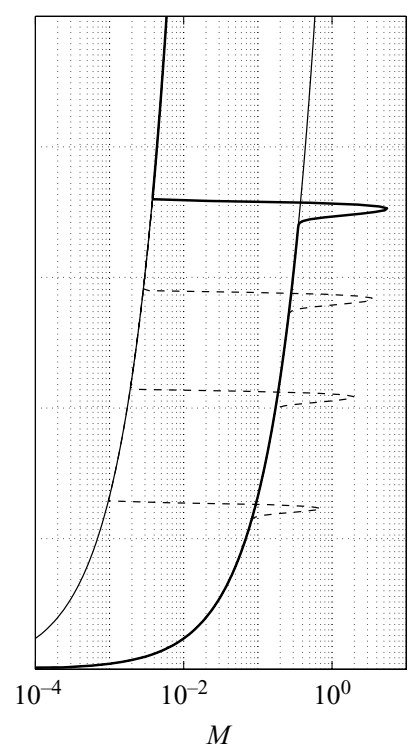

(c)

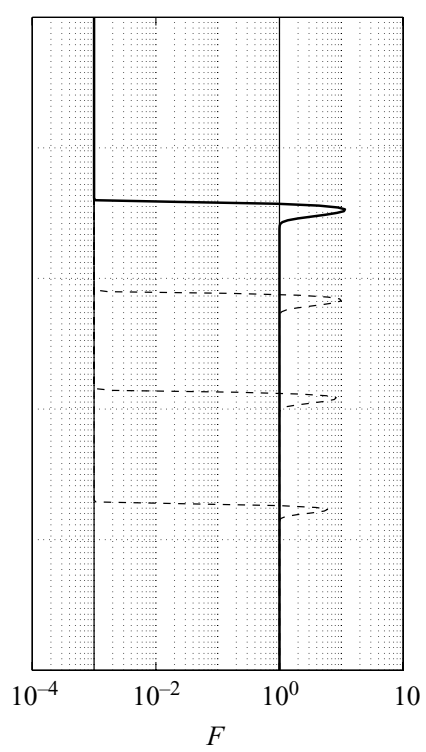

FIGURE 1. Typical plots of the evolution of the three primary dependent variables, $Q, M$ and $F$, against height $z$, as the buoyancy flux at the source has been increased from $F_{0}=10^{-3}$ to $F_{1}=1$. The solution at $t=0.5$ is shown as the thick solid line, the initial condition is the left-hand thin solid line in each image and the final state is the right-hand thin solid line in each image. Solutions at $t=0.13,0.25$ and 0.38 are shown as dashed lines. It can be seen that as the pulse propagates upwards the time-dependent solution adheres to the initial state solution above the pulse, but follows the final state solution below the pulse. The mass flux and momentum flux at the source remains zero throughout.

momentum flux at the source remains zero throughout. The boundary condition at the source is a step change in the buoyancy flux over one time step. Since backward differencing is used in the vertical direction, no boundary conditions are required at the top of the numerical box. The final states, shown as the right-hand thin solid line 


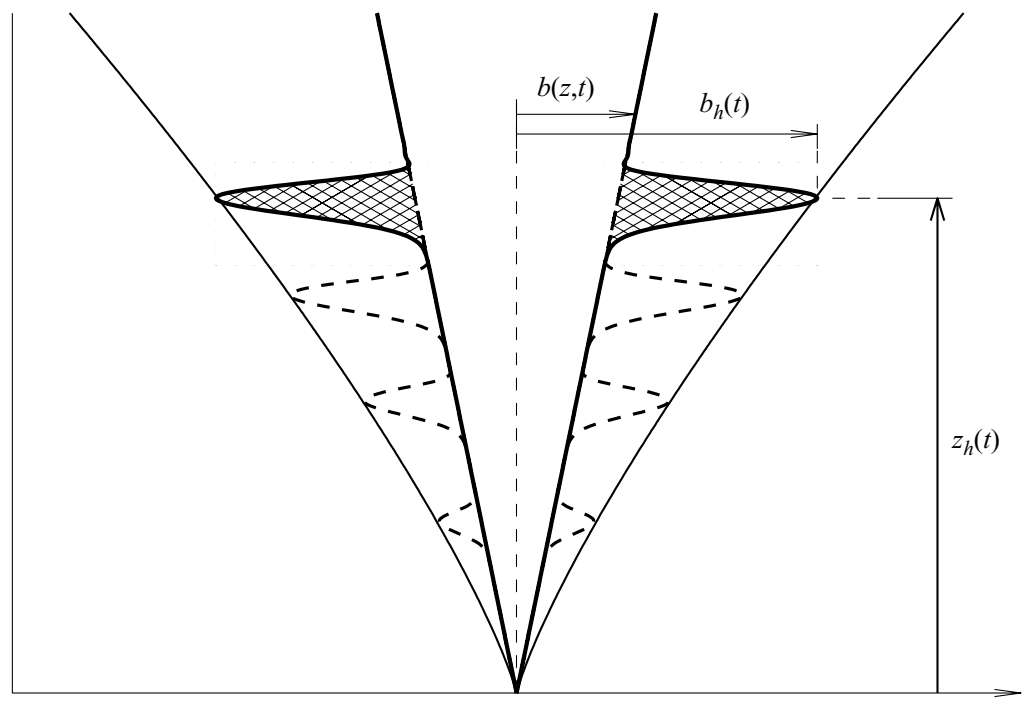

FIGURE 2. The evolution of a generic pulse propagating up a plume. The height of the pulse is denoted by $z_{h}(t)$. The extra volume entrained due to the pulse is found by rotating the hatched region about the axis of symmetry.

in each of the three images, are given respectively by

$$
Q=\left(\frac{5}{4}\right)^{1 / 3}\left(\frac{3 z}{5}\right)^{5 / 3}, \quad M=\left(\frac{5}{4}\right)^{2 / 3}\left(\frac{3 z}{5}\right)^{4 / 3}, \quad F=1 .
$$

The left-hand thin solid line in figure $1(a)$ is found by dividing $(2.2 a)$ by 10 , the left-hand thin solid line in figure $1(b)$ is found by dividing $(2.2 b)$ by $10^{2}$ and finally the left-hand thin solid line in figure $1(c)$ is found by dividing $(2.2 a)$ by $10^{3}$. It is seen that as the pulse propagates upwards the time-dependent solution adheres to the initial state solution above the pulse, but follows the final state solution below the pulse.

The fundamental behaviour of this system is shown in figure 2. Several time instants are shown. As was first demonstrated in Morton et al. (1956), the conical shape of a steady point source pure plume is independent of the source buoyancy flux. Hence, when an established plume is subjected to a rapid increase in its source buoyancy flux a new steady-state plume begins to establish itself near the source with the expected $b=3 z / 5$ plume profile first established in Morton et al. (1956). The propagation velocity of information about the change in source strength means that far away from the source the plume is as yet unaffected and so still retains its initial $b=3 z / 5$ plume profile. In between these near and far regions we observe the added feature of a bulging pulse propagating up the plume.

In the buoyancy decrease problem a transient region is formed which is narrower than the initial $b=3 z / 5$ profile. This narrower region extends over some nondegenerate height and is defined by the plume radius itself. Since this narrower region of interest is given by the plume radius it follows that the time-dependent equations can support similarity power-law solutions that determine the nature of the narrower region. On the other hand, in the buoyancy increase problem shown schematically in figure 2, such simple power-law solutions of the equations are not going to yield information about the evolution of the envelope of the plume radius 
(the thin solid line) as at a given time, the envelope only intersects the instantaneous solution to the time-dependent system at a single height, $z_{h}$ (a horizontal, circular intersection).

It will be shown that, to a good approximation, the pulse that is formed is selfsimilar not only with time in a given solution, but also across all seven source buoyancy flux increases considered. That is, the form of the pulse is self-similar across a range of seven orders of magnitude of buoyancy flux increase. Scaling laws for the extent of the pulse are established. The method of solution is as described in the Appendix of Scase et al. (2006b) except that in the present paper solutions were stepped forward in time using a fourth-order Runge-Kutta scheme.

\subsection{Comparison with the time-dependent model}

Figure 2 shows generic time shots of the evolution of the plume's radius. It is well known that the vertical velocity of a steady plume scales as $w_{\star} \sim F_{0 \star}^{1 / 3} z_{\star}^{-1 / 3}$ (see e.g. Zeldovich 1937; Morton et al. 1956) and so by increasing the strength of the source buoyancy flux we increase the vertical velocity of the plume fluid leaving the source (observing that exactly at the source this law predicts non-physical infinite velocities for all source buoyancy fluxes). This creates a region of fast-moving plume fluid with slower moving plume fluid ahead of it. As in the buoyancy decrease case, a steadystate plume's radius is independent of the source buoyancy flux and so after time a new fast-moving plume with radius $b=3 z / 5$ is established near to the plume source. Within the framework of top-hat plumes (or indeed any height-wise self-similar plume model) no mechanism exists for this faster moving plume fluid to be able to overtake the slower moving fluid ahead, and so a bulge appears in the plume radius (see figure 2). This bulge can be regarded as a 'pulse' which propagates up the plume. We denote the height of the maximum radius of this pulse at time $t$ by $z_{h}(t)$ and the maximum radius of the pulse at this time by $b_{h}(t)$, as marked in figure 2 .

The pulse rises up the plume, separating slower moving plume fluid ahead of the pulse from faster moving plume fluid behind the pulse. As the pulse rises the overall effect of fast-moving fluid entering the pulse from behind together with slower moving plume fluid ahead of the pulse and the entrainment of ambient fluid with no vertical momentum (see Scase et al. 2007) is to decrease the velocity of the pulse. It can also be observed that the pulse's volume (defined by rotating the hatched region in figure 2 azimuthally about the axis of symmetry) also increases.

The prediction of a bulging pulse within a top-hat plume model could manifest itself in two ways in a real plume. As described in Scase et al. $(2008, \S 5.3)$, in order to calculate an effective top-hat radius from a real plume, both the plume fluid concentration and plume radius must be combined. It does not therefore follow that a bulging pulse in the top-hat model will lead to an increase in a real plume's radius. In fact it was observed in the buoyancy reduction studies that almost all of the predicted narrowing in the top-hat model was accounted for by a reduction in plume fluid concentration at a given height, not in the standard deviation of a best Gaussian fit, which remained approximately linear.

For an ideal steady plume (in pure plume balance with a point source) obeying the Morton et al. (1956) model, there exists no length scale in the problem - the plume looks identical for all observers regardless of their horizontal distance from the source. For the case of the buoyancy increase described above, at time $t_{\star}$ a length scale is available, namely $z_{h \star}$. Hence, we may expect the volume of the pulse $V_{h \star}$ to scale as $V_{h \star} \sim z_{h \star}^{3}$ on dimensional grounds. 


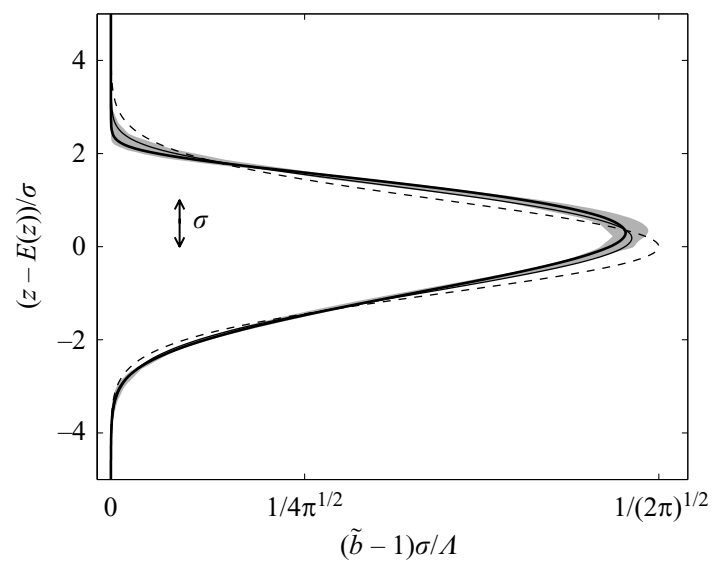

Figure 3. The self-similar form of the pulse; the thick solid line is the mean scaled and translated form of the pulse, the greyed area shows the maximum deviation from the mean. The dashed line is a Gaussian curve, for reference, and the thin solid line is the mean pulse form calculated from the lower resolution solutions.

If a tracer is introduced to the source of a steady ideal plume it is straightforward to show that the tracer reaches a height

$$
z_{\star}=\left(\frac{10}{9 \alpha^{(P)}}\right)^{1 / 2}\left(\frac{F_{0 \star}}{\pi \rho_{\infty \star}}\right)^{1 / 4} t_{\star}^{3 / 4}
$$

at some time $t_{\star}$ later (see e.g. Scase et al. 2006b, (5.3)). Equivalently, in non-dimensional terms, $z=(20 / 9)^{1 / 2} t^{3 / 4}$. Guided by the result in (2.3), and on dimensional grounds, we therefore expect the pulse height to scale as $z_{h \star} \sim t_{\star}^{3 / 4}$ and the pulse volume to scale as $V_{h \star} \sim t_{\star}^{9 / 4}$.

We translate and scale $b$ to show the self-similarity of the pulse over a large range of buoyancy increases and times. We define the following quantities:

$$
\Lambda_{\star}=\int_{0}^{\infty}(\tilde{b}-1) \mathrm{d} z_{\star}, \quad E\left(z_{\star}\right)=\frac{1}{\Lambda_{\star}} \int_{0}^{\infty}(\tilde{b}-1) z_{\star} \mathrm{d} z_{\star}, \quad E\left(z_{\star}^{2}\right)=\frac{1}{\Lambda_{\star}} \int_{0}^{\infty}(\tilde{b}-1) z_{\star}^{2} \mathrm{~d} z_{\star},
$$

where $\tilde{b}=5 b /(3 z)$. The quantity $\tilde{b}$ may thus be thought of as the fractional deviation of the actual plume radius from the steady solution, $b=3 z / 5$. Equation (2.4) leads to a natural and robust definition of a vertical length scale $\sigma_{\star}$ given by

$$
\sigma_{\star}=\left\{E\left(z_{\star}^{2}\right)-E\left(z_{\star}\right)^{2}\right\}^{1 / 2}
$$

It follows that $\Lambda_{\star} / \sigma_{\star}$ is therefore a measure of the horizontal fractional deviation from the steady plume radius, and so we take $\Lambda_{\star} z_{h_{\star}} / \sigma_{\star}$ as a measure of the horizontal extent of the pulse. The quantities $\Lambda_{\star}$ and $E\left(z_{\star}\right)$ have dimensions of length and $E\left(z_{\star}^{2}\right)$ has dimensions of length squared (their non-dimensional counterparts are given by $\Lambda=\Lambda_{\star} / z_{\ell_{\star}}, E(z)=E\left(z_{\star}\right) / z_{\ell_{\star}}$ and $E\left(z^{2}\right)=E\left(z_{\star}^{2}\right) / z_{\ell_{\star}}^{2}$ ). Figure 3 shows the rescaled pulses for the seven different buoyancy increases. The thick black solid line is the mean across all times and all buoyancy increases, the greyed area shows the maximum deviation from the mean for all times and all buoyancy increases. There is an extremely small deviation from the mean. The thin solid line shows the mean from a low-resolution solution (see appendix B for further discussion of low-resolution solutions), demonstrating that the self-similar shape is independent 
of resolution to a good approximation. The dashed line shows the Gaussian curve $\exp \left\{-[z-E(z)]^{2} /\left(2 \sigma^{2}\right)\right\} / \sqrt{2 \pi}$ for reference. Comparison between the mean pulse shape and the Gaussian curve shows that the pulse is slightly skewed towards the top of the plume, i.e. the pulse is negatively skewed. If the pulse was symmetric $E(z)$ would equal $z_{h}(t)$, but in fact, due to the skewness, $E(z)<z_{h}(t)$.

The self-similarity of the pulse over such a large range of buoyancy increases, and the fact that it persists is perhaps remarkable. It is certainly encouraging as it offers the opportunity for a general description of the effects of buoyancy increases, as was found for buoyancy decreases, although the methodology must differ for the reasons described in $\S 2.1$.

Figure 4(a) shows the evolution of the rise height of the maximum amplitude of the pulse, $z_{h}(t)$. The legend indicates which buoyancy increase corresponds to which data points. For clarity on the graphs the data points for each data set are separated by non-dimensional time 1.4 and each data set is staggered in steps of $t=0.2$, i.e. only seven data points from a set of $2.5 \times 10^{5}$ have been plotted for each of the seven solutions. The data points for the different buoyancy increases were best fitted to a $\lambda^{\prime} t^{3 / 4}$ curve (where $\lambda^{\prime}$ depends on $F_{0}$ and $F_{1}$ ) on a $\log -\log$ plot and have been rescaled by $1 / \lambda^{\prime}$ in $(b)$ so that, for a perfect fit, all the data points would sit exactly on the $t^{3 / 4}$ line. The values of $\lambda^{\prime}$ are reported in table 1. Figure 4(a) supports the prediction of a $t^{3 / 4}$ scaling for the rise height of the pulse across a large number of orders of magnitude of buoyancy increase.

Figure $4(b)$ shows the evolution of the volume of the pulse $V_{h}(t)$. The same method of scaling and plotting as used in $(b)$ has been repeated except that for a perfect fit all the data points would sit exactly on the $t^{9 / 4}$ line. As discussed above, it is perhaps expected that since the length scale $z_{h} \sim t^{3 / 4}$, the volume of the pulse scales as $V_{h} \sim t^{9 / 4}$.

The scalings of the rise height and volume of the pulse are consistent with the existing scaling laws for steady plume behaviour, as described above (see (2.3)). However, figure $4(c, d)$ shows that there exist vertical and horizontal length scales for the pulse which have $t^{1 / 4}$ and $t$ scalings, respectively.

Figure 5 shows the typical variation of the plume's vertical velocity $w$ and reduced gravity $g^{\prime}$ with height $z$. It can be seen that there is a sharp transition in both quantities at the height of the pulse $z_{h}$. Figure 5(a) shows the vertical velocity field against height for a plume that has been subjected to a change in source strength from $F_{0}=10^{-2}$ to $F_{1}=1$ at a time $t=0.38$ after the increase. The height $z=z_{h}$ is calculated from the values given in table 1 giving $z_{h}=0.60$ at $t=0.38$. Information about the change in source strength has not yet propagated above the line $z=z_{h}$, and so the data points sit on the curve

$$
w(z)=\frac{5}{3}\left(\frac{9}{20}\right)^{1 / 3} F_{0}^{1 / 3} z^{-1 / 3},
$$

which is the left-hand dashed line in figure $5(a)$. Below the line $z=z_{h}$ the plume has adjusted to its new source strength and so the data points sit on the curve (2.6) with $F_{0}$ replaced by $F_{1}$ (the right-hand dashed line in figure $5(a)$ ).

Figure $5(b)$ is the corresponding reduced gravity field to plot $(a)$. Above the line $z=z_{h}$ the data points sit on the curve

$$
g^{\prime}(z)=\frac{5}{3}\left(\frac{20}{9}\right)^{1 / 3} F_{0}^{2 / 3} z^{-5 / 3},
$$


(a)

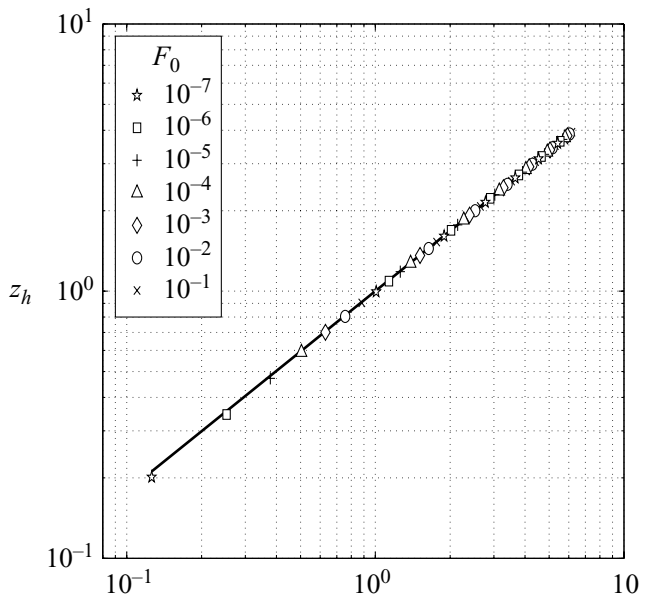

(c)

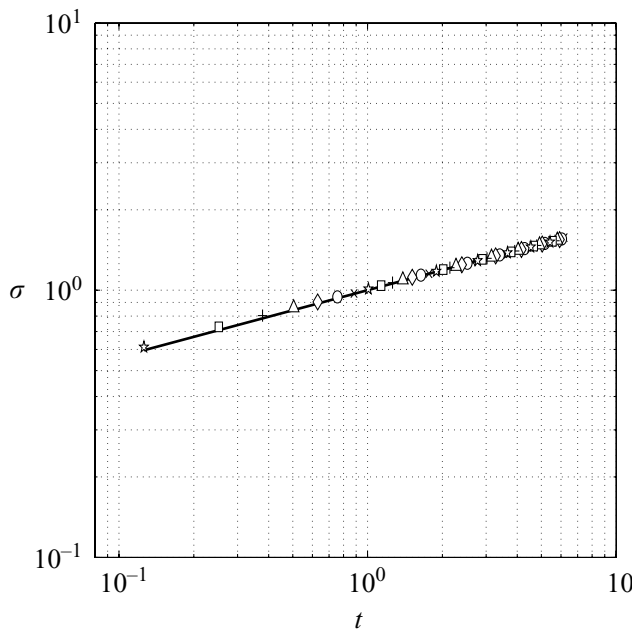

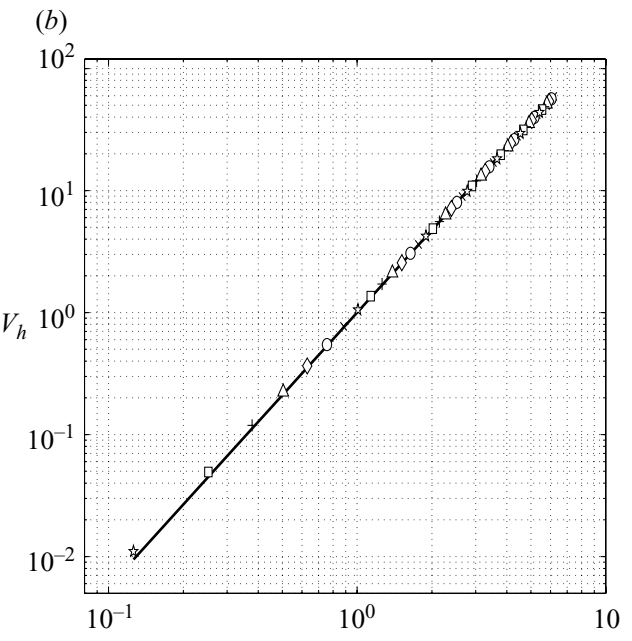

(d)

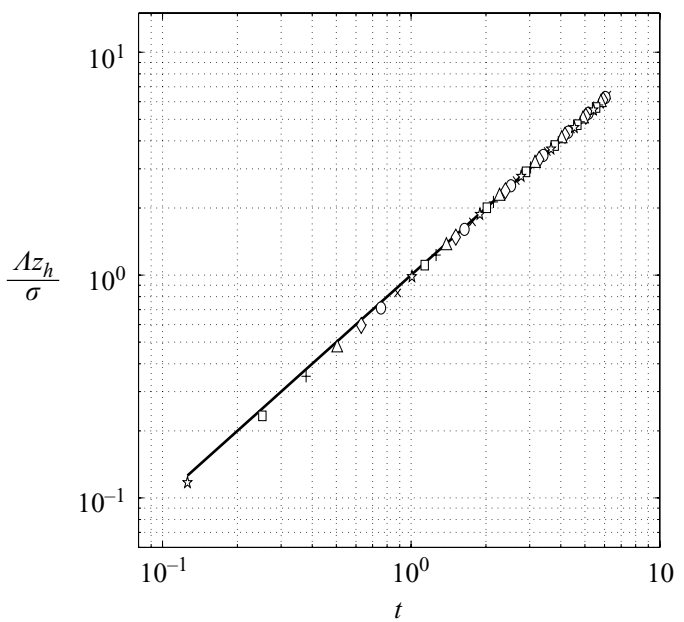

FIGURE 4. For the seven different buoyancy increases considered: $(a)$ shows the scaled evolution of the pulse's height, $z_{h}(t)$, and the solid line is $t^{3 / 4} ;(b)$ shows the scaled evolution of the pulse's volume, $V_{h}(t)$, the solid line is $t^{9 / 4} ;(c)$ shows the scaled evolution of the pulse's vertical length scale, $\sigma$, the solid line is $t^{1 / 4} ;(d)$ shows the scaled evolution of the pulse's horizontal length scale, $\Lambda z_{h} / \sigma$, the solid line is linear $t$. The legend in $(a)$ also applies to the plots in $(b)-(d)$.

which is the left-hand dashed line in $(b)$. Below the line $z=z_{h}$ the data points sit on the curve (2.7) with, again, $F_{0}$ replaced by $F_{1}$ (the right-hand dashed line in figure $5(b))$. The sharp transition in both $(a)$ and $(b)$ is due to the small vertical extent $\sigma$ of the pulse.

\subsection{Distributed source plumes}

Real-life turbulent jets and plumes originate from non-point sources, and in that sense we refer to their sources as 'distributed' (Caulfield \& Woods 1995) since the source is distributed over an area. Very often a plume with a distributed source has large source buoyancy and mass fluxes compared to its source momentum flux in 
(a)

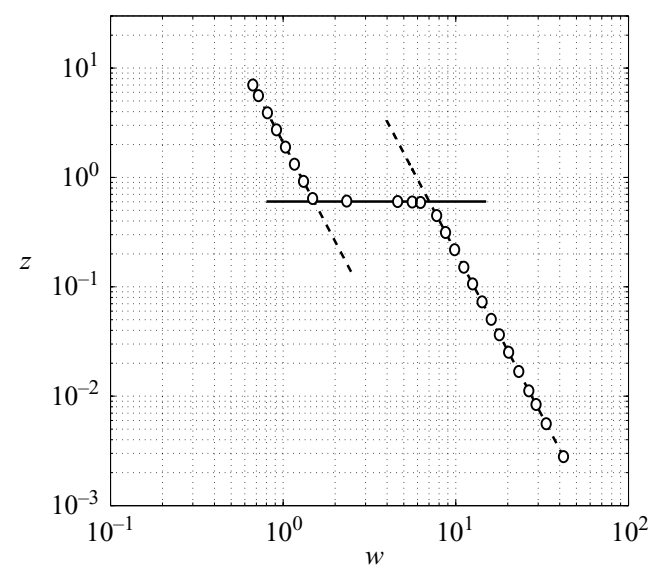

(b)

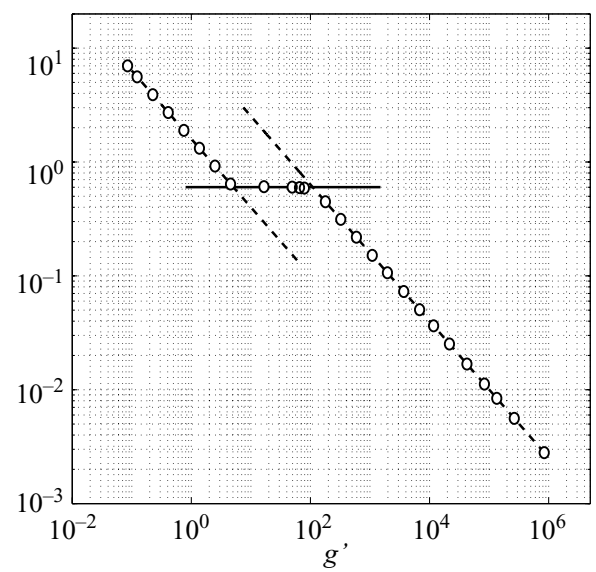

FIGURE 5. (a) The variation of the vertical velocity $w$ in the plume after the plume has been subjected to an increase in source strength from $F_{0}=10^{-2}$ to $F_{1}=1$. The dashed lines follow the usual $z^{-1 / 3}$ power law. The solid line is $z=z_{h}$, where $z_{h}$ has been calculated using the constants given in table 1. (b) The variation of the reduced gravity $g^{\prime}$ in the plume after the plume has been subjected to the same increase in source strength as in $(a)$. The dashed lines follow the usual $z^{-5 / 3}$ power law. The solid line is as in $(a)$. (Not all data points are shown.)

the sense that the plume's source laziness $\Gamma_{0}$ is greater than unity. Such plumes have become known as 'lazy' (Hunt \& Kaye 2001) since, equivalently, they may be thought of as plumes with a deficiency of momentum flux compared to a pure plume. Not all plumes with distributed sources are necessarily lazy; in the present section we consider pure plumes $\left(\Gamma_{0}=1\right)$ originating from a distributed source.

A common approach to modelling distributed source plumes emanating from a horizontal circular source of radius $b_{0 \star}$ is to consider a 'virtual-origin' correction, whereby the plume behaviour far from the source, $z_{\star} \gg b_{0 \star} /\left(2 \alpha^{(P)}\right)$, is as if the plume originated from a point source located at a virtual origin situated at some $z_{\star}=-z_{v s \star}$. For a plume in pure plume balance it is straightforward to show that $z_{v s \star}=5 b_{0 \star} /\left(6 \alpha^{(P)}\right)$ by means of a 'conical correction' (Caulfield 1991; Caulfield \& Woods 1995; Hunt \& Kaye 2001). For non-pure plumes with $\Gamma_{0} \neq 1$ the value of $z_{v s \star}$ becomes harder to find and is a function of both $b_{0 \star}$ and $\Gamma_{0}$ (see Hunt \& Kaye 2001, Scase et al. 2008). For a pure plume originating at a distributed source, all plume quantities develop in pure plume balance immediately from the source with no region of adjustment. However, the situation is qualitatively different for time-dependent problems.

The introduction of a length scale $b_{0 \star}$ is important when considering buoyancy increases to plumes. In $\S 2.2$ it was shown that the volume of a pulse formed due to a buoyancy increase at the source of an established plume scaled as the rise height of the pulse to the third power. It was suggested that this might be expected since $z_{h \star}$ is the natural length scale. With the introduction of an imposed length scale on the system this dimensional argument may no longer apply for all heights, however, far from the source it must still be expected that the scaling laws established in $\S 2.2$ can be applied.

Figures 6 and 7 show the results of four solutions to the time-dependent plume equations. The numerical box used for the solutions is the same as in $\S 2.2$, as was the resolution employed. In each of the four solutions the buoyancy flux of an established plume was increased from an initial value of $F_{0}=10^{-2}$ to a final value $F_{1}=1$. Four 


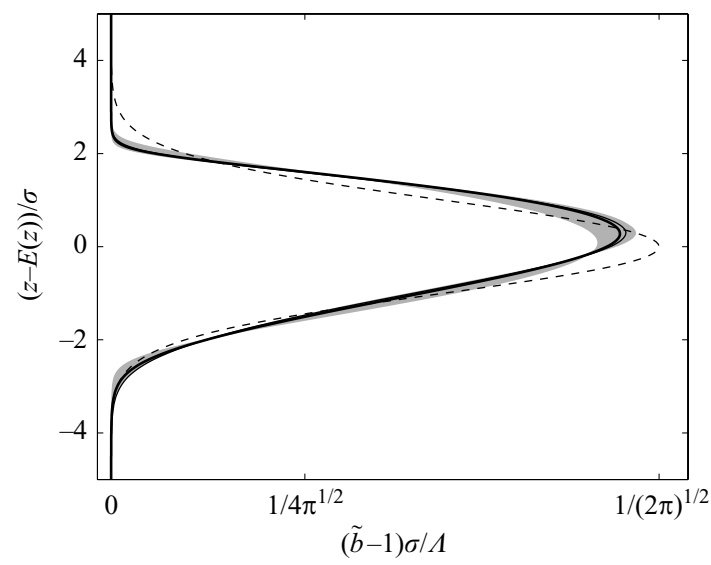

FiguRE 6. The self-similar pulse shape for the different source sizes. The thick solid line is the average across all source sizes and times, the greyed area show the maximum deviation of the individual source size mean profiles. The thin solid line is the mean point source pulse profile from figure 3 .

different source sizes were considered, $b_{0}=10^{-2}, b_{0}=10^{-1}, b_{0}=10^{0}$ and $b_{0}=10^{1}$, where $z_{\ell \star}=1 \mathrm{~m}$.

Figure 6 shows again that the shape of the pulse remains to a good approximation self-similar across three orders of magnitude of source size. The dashed line is a reference Gaussian curve and the thin solid line is the mean pulse shape from a point source plume taken from figure 3 (note that this is a mean across all seven buoyancy flux increases considered in $\S 2.2$, not just $F_{0}=10^{-2}$ to $F_{1}=1$ considered here). The thick dashed line is the mean pulse shape for all four source sizes considered, the greyed area shows the maximum deviation of the individual source size means from the overall mean. As for the point source plumes considered in $\S 2.2$ the pulse shape is again negatively skewed towards the top of the plume.

It follows from the non-dimensionalization in (1.5) that the introduction of a length scale $z_{\ell \star}$ automatically introduces a time scale

$$
t_{\ell \star}=\left(4 \alpha^{(P) 2} \pi \rho_{\infty \star}\right)^{1 / 3} F_{0 \star}^{-1 / 3} z_{\ell \star}^{4 / 3} .
$$

Therefore, for a given length scale $z_{\ell_{\star}}$, a plume emanating from a large source $b_{0 \star} /\left(2 \alpha^{(P)} z_{\ell_{\star}}\right) \gg 1$ may equivalently be thought of as the early time evolution of a plume emanating from a source $b_{0 \star} /\left(2 \alpha^{(P)} z_{\ell \star}\right)=1$. Similarly a plume emanating from a small source $b_{0_{\star}} /\left(2 \alpha^{(P)} z_{\ell_{\star}}\right) \ll 1$ may equivalently be thought of as the late time evolution of a plume emanating from a source $b_{0 \star} /\left(2 \alpha^{(P)} z_{\ell \star}\right)=1$. From this observation we note that the point source scaling laws found in $\S 2.2$ can be considered as limiting scaling laws for the long-time behaviour of plumes from distributed sources.

Figure 7(a) shows the rise height of the pulse on a log-log plot with the nondimensional lengths now rescaled by $b_{0}$ and the non-dimensional times rescaled by $b_{0}^{4 / 3}$. The four solutions for the differing source sizes all dovetail onto the same curve. Applying a rescaling of the non-dimensional steady Morton et al. (1956) equations, based on the source radius, yields a rise height, equivalent to (2.3), given by

$$
z_{h}=\frac{5}{3}\left\{\left[\left(\frac{4}{5}\right)^{2 / 3} t+1\right]^{3 / 4}-1\right\} .
$$


(a)

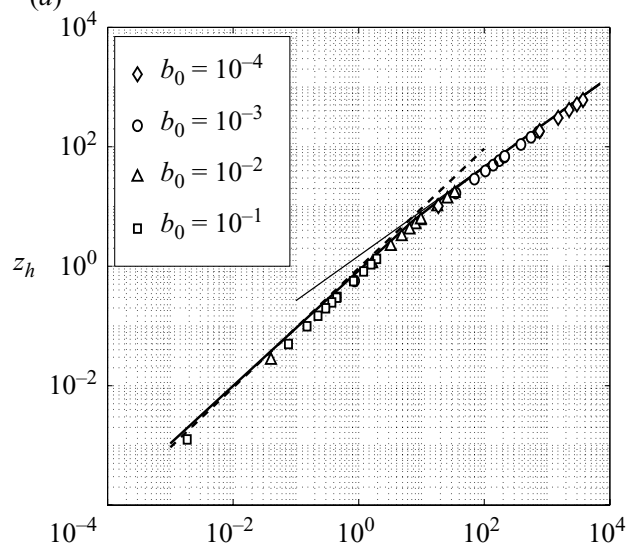

(c)

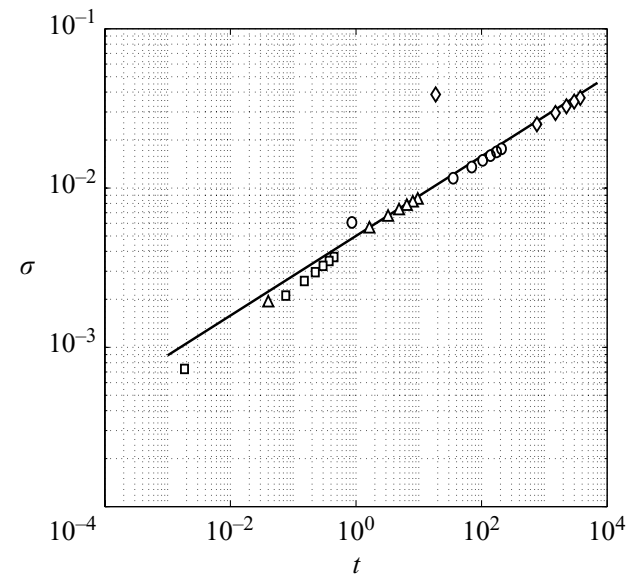

(b)

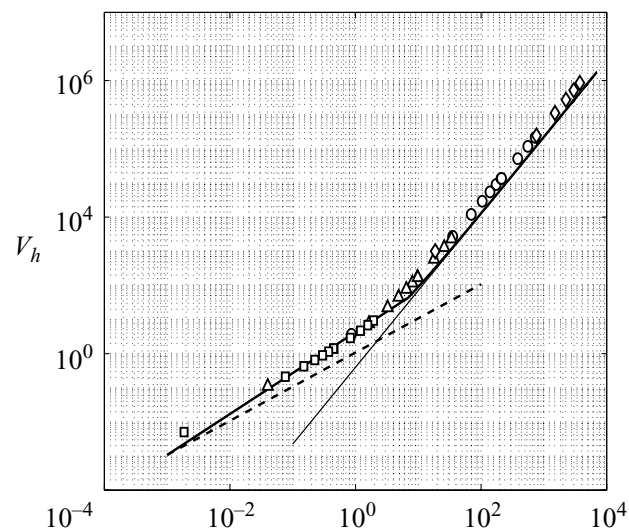

(d)

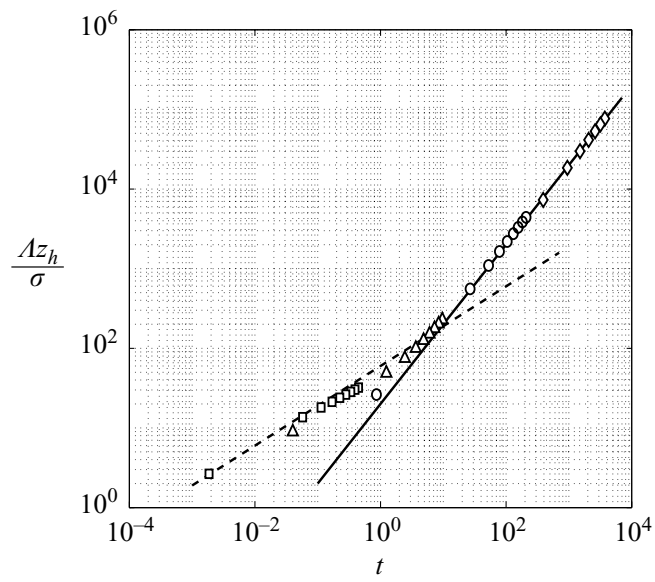

FIGURE 7. (a) The change in scaling for the rise height of the pulse for a distributed source plume against time. The thick solid line is given by (2.9). The thin solid line is $z=(20 / 9)^{1 / 2} t^{3 / 4}$ and the dashed line is $z=(4 / 5)^{1 / 3} t$. $(b)$ The change in scaling for the volume of the pulse for a distributed source plume against time. The thick solid line is given by (2.10). The thin solid line is $z=(4 / 9)(4 / 5)^{1 / 2} t^{9 / 4}$ and the dashed line is $z=(5 / 4)^{1 / 3} t$. (c) The scaling for the height of the pulse for a distributed source plume against time. The solid line is proportional to $t^{1 / 4}$ but the different rescaling, $b_{0}^{1 / 3}$, for the length $\sigma$ must be used. $(d)$ The scaling for the horizontal extent of the pulse for a distributed source plume against time. The solid line is linear in $t$ and the dashed line is proportional to $t^{1 / 2}$. The quantity $\Lambda z_{h} / \sigma$ has been rescaled by $b_{0}^{4 / 3}$.

This curve (2.9) is shown as the thick solid line in figure $7(a)$. It can be seen from (2.9) that for small $t \ll 1$ the rise height is asymptotically linear in $t$ with $z_{h} \sim(5 / 4)^{1 / 3} t+O\left(t^{2}\right)$. This linearity is because the velocity near to the source is dominated by the initial velocity, and convective and entrainment effects have not yet had a significant effect. For large $t \gg 1$ the rise height in (2.9) is asymptotically given by $z_{h} \sim(20 / 9)^{1 / 2} t^{3 / 4}+O(1)$, i.e. the rise height is asymptoting towards the same rise height as if the plume had originated from a point source. This is because far from the source the plume's behaviour is dominated by the entrainment and convection along the plume's length and the exact conditions at the source can only have an $O(1)$ effect (analogously to a virtual origin correction). The thin solid line 
in figure $7(a)$ follows a $t^{3 / 4}$ power law and it can be seen that as time increases the power law dependence of each of the four solution sets tends towards this $t^{3 / 4}$ scaling. Furthermore, the data set with the smallest source size $\left(b_{0}=10^{-2}\right.$ indicated by diamonds) which, dimensionally, is most similar to the point source solution of $\S 2.2$, and can be considered equivalently as the late time solution of a generic distributed source with unit source radius, follows the $t^{3 / 4}$ scaling closely. The data also suggest that the early time scaling for the rise height of the pulse indeed goes linearly in $t$.

Figure $7(b)$ shows the evolution of the volume of the pulse, as defined in $\S 2.2$. We make the approximation

$$
V_{h} \approx \int_{0}^{t} Q\left[z_{h}(t)\right] \mathrm{d} t=\frac{5}{9}\left\{\left[\left(\frac{4}{5}\right)^{2 / 3} t+1\right]^{9 / 4}-1\right\},
$$

and curve (2.10) is shown as the thick solid line. The thin solid line follows a $t^{9 / 4}$ scaling and the dashed line follows in linear scaling in $t$. The data point symbols are as in figure $7(a)$. Again, the data have been non-dimensionalized, by $4 \pi \alpha^{(P)} 2 z_{\ell_{\star}}^{3}$ for the volume and (2.8) for the time, and rescaled to fit onto a single curve. As before, the late time behaviour, far from the source, follows the point source plume scaling of $V_{h} \sim t^{9 / 4}$. This can be predicted from (2.10) since for large $t, V_{h} \sim(4 / 9)(4 / 5)^{1 / 2} t^{9 / 4}+O\left(t^{5 / 4}\right)$. However, the effect of the introduction of the length $b_{0}$ is to force an early time, near source, scaling of the volume that is linear in $t$. From (2.10), for small $t$, $V_{h} \sim(5 / 4)^{1 / 3} t+O\left(t^{2}\right)$, as for the pulse rise height.

Figure 7(c) shows the evolution of the vertical extent of the pulse $\sigma$. The data point symbols are as in figure $7(a)$. In $\S 2.2$ it was shown that although the height $z_{h} \sim t^{3 / 4}$ and the volume of the pulse $V_{h}$ therefore scaled as $V_{h} \sim t^{9 / 4}$, the vertical extent of the pulse scaled as $\sigma \sim z_{h}^{1 / 3} \sim t^{1 / 4}$. In figure $7(c)$ the time has been non-dimensionalized by (2.8) and the solid line follows a $t^{1 / 4}$ scaling. Since the vertical extent of the pulse scaled as $z_{h}^{1 / 3}$ for the point source plume, here the vertical extent of the pulse has been scaled by $b_{0}^{1 / 3}$. As in figure $7(a, b)$, the data points dovetail onto the same curve and reveal that there is no significant change in the scaling in the vertical extent of the pulse as regards early or late times, or near or far source distances. Figure $7(d)$ demonstrates that initially $\Lambda z_{h} / \sigma$ follows a $t^{1 / 2}$ before reverting to the linear point source $t$ scaling, where $\Lambda_{\star}$ is defined as in (2.4).

\subsection{Discussion}

The vertical velocity of a mass of plume fluid exiting from a distributed source in pure plume balance has three factors contributing to its evolution. The fluid experiences acceleration due to its density difference with its surroundings. The fluid also experiences some deceleration due to the entrainment of ambient fluid which has no vertical momentum. Near the distributed source, however, since its velocity is dominated by the initial velocity required to keep the plume in pure plume balance, the fluid has some inertia due to its mass, unlike a point source plume. So, for early times, near the plume source, since the velocity is dominated by its initial value, and thus remains approximately constant, before any significant acceleration or deceleration can take place, it follows that the rise height $z_{h}$ scales linearly with time $t$. Similarly to the vertical velocity, the mass flux near a distributed source is dominated by the initial value required to keep the plume in pure plume balance. Hence, when the source strength is increased there is an excess mass flux $Q_{1_{\star}}-Q_{0_{\star}}$ and the volume of the pulse therefore initially evolves as $\left(Q_{1_{\star}}-Q_{0_{\star}}\right) t_{\star} / \rho_{\infty \alpha_{\star}}$. The reason for the $t^{1 / 4}$ scaling for the vertical extent of the pulse, $\sigma$, is perhaps less clear, as is the reason that $\sigma$ 
seems unaffected by whether the plume originates at a point source or a distributed source.

\section{Numerical modelling of turbulent plumes}

\subsection{Introduction}

Three-dimensional numerical simulations were conducted using a publicly available incompressible Navier-Stokes solver (IAMR Center for Computational Sciences and Engineering, Lawrence Berkeley National Laboratory: http://seesar. lbl.gov/CCSE/index.html). IAMR employs a finite-volume approach on a threedimensional Cartesian mesh with a two-step predictor-corrector method based on the unsplit second-order Godunov methodology introduced for gas dynamics by Colella (1990). The advective velocities are constructed using a monotonicity-limited fourth-order centred-difference slope approximation (Colella 1985). An intermediate marker-and-cell (MAC) projection (Bell, Colella \& Howell 1991) is used to ensure these velocities are discretely divergence free before the flow variables are advected. Finally, an approximate projection (Almgren, Bell \& Crutchfield 2000) is used to enforce the divergence-free constraint in the updated velocity field. IAMR utilizes adaptive mesh refinement to focus resolution where it is required, significantly reducing computational expense. The code is also parallelized and scales well to many thousands of processors. The overall algorithm is second-order in both space and time. For further details see Almgren et al. (1998) and the references therein.

IAMR is capable of implicit large eddy simulations (ILES), which use nonoscillatory finite-volume schemes to capture the inviscid cascade of kinetic energy through the inertial range, where small-scale motions, larger than the Kolmogorov length scale, behave inviscidly and their evolution depends solely upon the rate of energy dissipation. The inherent numerical dissipation acts as an implicit subgrid model meaning that no extra modelling, or explicit turbulence closure scheme, is required. The approach was introduced by Boris (1990) (see also Boris et al. 1992), and is becoming widely used for many applications (for examples, see Youngs 1991; Porter, Pouquet \& Woodward 1992; Fureby \& Grinstein 1999; Margolin, Rider \& Grinstein 2006; Drikakis et al. 2007). An overview of the technique, including a historical background and other applications, can be found in the book by Grinstein, Margolin \& Rider (2007). Aspden et al. (2008) used simulations of homogeneous isotropic turbulence to investigate the performance of IAMR for ILES, and proposed a methodology for characterizing its behaviour. It was demonstrated that for fully developed turbulence, the effective Kolmogorov length scale was approximately 0.3 times the computational cell width, making the approach significantly less expensive than direct numerical simulation.

\subsection{The numerical scheme}

The equations of motion solved by IAMR for the present paper are the incompressible Euler equations, with buoyancy described by a temperature perturbation under the Boussinesq approximation. Specifically

$$
\begin{gathered}
\frac{\mathrm{D} \boldsymbol{u}_{\star}}{\mathrm{D} t_{\star}}=-\nabla_{\star} P_{\star}+g_{\star} \beta_{\star}\left(T_{\star}-T_{\infty \star}\right) \boldsymbol{e}_{z}, \\
\frac{\mathrm{D} T_{\star}}{\mathrm{D} t_{\star}}=0, \quad \nabla_{\star} \cdot \boldsymbol{u}_{\star}=0 .
\end{gathered}
$$


The buoyancy acceleration in (3.1) is expressed in terms of a temperature $T_{\star}$, reference temperature $T_{\infty \star}$ and thermal expansion coefficient $\beta_{\star}$. The modified pressure is given by $P_{\star}=p_{\star} / \rho_{\infty \star}-g_{\star} z_{\star}$.

The plume source was modelled by a mass, momentum and buoyancy inflow through a 'circular nozzle' in the centre of the base of the solution domain. The velocity at the source was perturbed using a three-dimensional velocity field output from a homogeneous isotropic turbulence simulation. Transmissive lateral boundary conditions were used to allow fluid to be drawn into the domain horizontally and enable entrainment without creating large recirculation regions. The upper boundary condition was also transmissive, with negative vertical velocities prohibited. Adaptive mesh refinement was used to reduce computational expense. The grid was refined around areas of high temperature gradient, where vorticity is generated due to baroclinic instability. A base Cartesian grid of $128 \times 128 \times 192$ zones was used, with two levels of refinement giving an effective resolution of $512 \times 512 \times 768$. Refinement was restricted to the lower $80 \%$ of the domain, making a buffer zone at the top of the domain to minimize the effect of the upper boundary condition. The nozzle was resolved by eight cells across the diameter, which makes the domain $64 \times 64 \times 96$ nozzle diameters in size.

The numerics were run to simulate the model description in $\S 2$ as closely as possible. An initial turbulent buoyant plume was established, then the source strength was instantaneously increased. A simulation with statistically steady source conditions was run to provide initial conditions for the 'time-dependent' simulations. The classical steady-state plume was achieved after approximately 2500 iterations. The ensemble statistics were calculated from 12 separate realizations, where each realization was azimuthally averaged. The azimuthal averaging was achieved in the following manner. The data was interpolated onto a single-level fine grid with resolution $1024 \times 1024 \times 768$ using a standard central differencing scheme. In each of the 768 horizontal planes each data point was assigned an associated horizontal radial distance from the vertical $r=0$ axis. The $1024 \times 1024$ data points within the horizontal plane were then partitioned into 256 cells according to their associated radius. The partitioned cell value was then assigned to be the arithmetic mean of all its members. This process was repeated for each of the 768 horizontal planes yielding a two-dimensional azimuthal average of size $256 \times 768$.

The initial conditions for the time-dependent simulations were taken from the steady-state solution at intervals of 500 iterations. Each realization was run for 800 iterations, and data points output every 100 iterations. The time step at each iteration was set dynamically, controlled by the Courant-Friedrichs-Lewy (CFL) condition, but was approximately $0.064 \mathrm{~s}$. The CFL number in the presented simulations was 0.7 . The physical dimensions of the solution domain were $1.024 \mathrm{~m} \times 1.024 \mathrm{~m} \times 1.568 \mathrm{~m}$ with a plume source diameter $\varnothing_{\star}=0.016 \mathrm{~m}$. The fluid density was taken as $\rho_{\infty \star \star}=1000 \mathrm{~kg} \mathrm{~m}^{-3}$, the acceleration due to gravity was taken as $g_{\star}=9.81 \mathrm{~m} \mathrm{~s}^{-2}$, the thermal expansion coefficient was taken as $\beta_{\star}=2.1 \times 10^{-4} \mathrm{~K}^{-1}$ and the reference temperature was taken as $T_{\infty \star}=293.00 \mathrm{~K}$. The initial conditions prescribed at the source, prior to the source strength increase, were the vertical velocity $w_{0 \star}=0.024 \mathrm{~m} \mathrm{~s}^{-1}$ and temperature $T_{0 \star}=294.00 \mathrm{~K}$. After the source strength was increased, $w_{1 \star}=0.065 \mathrm{~m} \mathrm{~s}^{-1}$ and $T_{1 \star}=300.37 \mathrm{~K}$. The boundary conditions in terms of mass, momentum and buoyancy fluxes are described in table 2 . These boundary conditions gave an initial and final plume laziness at the source of $\Gamma_{0}=0.26$, a forced (or 'jetty') plume. The effective top-hat radius was calculated using the method described in Scase et al. $(2008, \S 5.3)$. 


\begin{tabular}{|c|c|c|c|c|c|c|c|}
\hline \multicolumn{2}{|c|}{ General simulation values } & \multicolumn{3}{|c|}{ Non-dimensional initial and final values } & \multicolumn{3}{|c|}{ Dimensional simulation values } \\
\hline $\begin{array}{l}\text { Plume nozzle } \\
\text { diameter }\end{array}$ & $\varnothing_{\star}=1.6 \times 10^{-2} \mathrm{~m}$ & Mass flux & $Q_{0}=0.62$ & $Q_{1}=1.69$ & Mass flux & $Q_{0 \star}=4.82 \times 10^{-3} \mathrm{~kg} \mathrm{~s}^{-1}$ & $Q_{1 \star}=1.31 \times 10^{-2} \mathrm{~kg} \mathrm{~s}^{-1}$ \\
\hline $\begin{array}{c}\text { Reference } \\
\text { density }\end{array}$ & $\rho_{\infty \star \star}=1000 \mathrm{~kg} \mathrm{~m}^{-3}$ & Momentum flux & $M_{0}=0.39$ & $M_{1}=2.86$ & Momentum flux & $M_{0 \star}=1.16 \times 10^{-4} \mathrm{~kg} \mathrm{~m} \mathrm{~s}^{-2}$ & $M_{1 \star}=8.49 \times 10^{-4} \mathrm{~kg} \mathrm{~m} \mathrm{~s}^{-2}$ \\
\hline $\begin{array}{l}\text { Reference } \\
\text { temperature }\end{array}$ & $T_{\infty \star}=273.00 \mathrm{~K}$ & Buoyancy flux & $F_{0}=0.05$ & $F_{1}=1.00$ & Buoyancy flux & $F_{0 \star}=1.01 \times 10^{-5} \mathrm{~kg} \mathrm{~m} \mathrm{~s}^{-3}$ & $F_{1 \star}=2.02 \times 10^{-4} \mathrm{~kg} \mathrm{~m} \mathrm{~s}^{-3}$ \\
\hline $\begin{array}{l}\text { Thermal } \\
\text { expansion }\end{array}$ & $\beta_{\star}=2.1 \times 10^{-4} \mathrm{~K}^{-1}$ & Laziness & $\Gamma_{0}=0.26$ & $\Gamma_{1}=0.26$ & $\begin{array}{l}\text { Plume source } \\
\text { temperature }\end{array}$ & $T_{0 \star}=294.00 \mathrm{~K}$ & $T_{1 \star}=300.37 \mathrm{~K}$ \\
\hline \multirow[t]{2}{*}{$\begin{array}{l}\text { Gravitational } \\
\text { acceleration }\end{array}$} & $g_{\star}=9.81 \mathrm{~m} \mathrm{~s}^{-2}$ & $\begin{array}{l}\text { Reynolds } \\
\text { number }\end{array}$ & $R e_{0}=384$ & $R e_{1}=1040$ & $\begin{array}{l}\text { Plume source } \\
\text { velocity }\end{array}$ & $w_{0 \star}=2.4 \times 10^{-2} \mathrm{~m} \mathrm{~s}^{-1}$ & $w_{1 \star}=6.5 \times 10^{-2} \mathrm{~m} \mathrm{~s}^{-1}$ \\
\hline & & $\begin{array}{l}\text { Solution } \\
\text { domain }\end{array}$ & $512 \times 512 \times$ & 678 & $\begin{array}{l}\text { Solution } \\
\text { domain }\end{array}$ & $1.024 \mathrm{~m} \times 1.024 \mathrm{~m} \times 1.568 \mathrm{~m}$ & \\
\hline
\end{tabular}


(a)

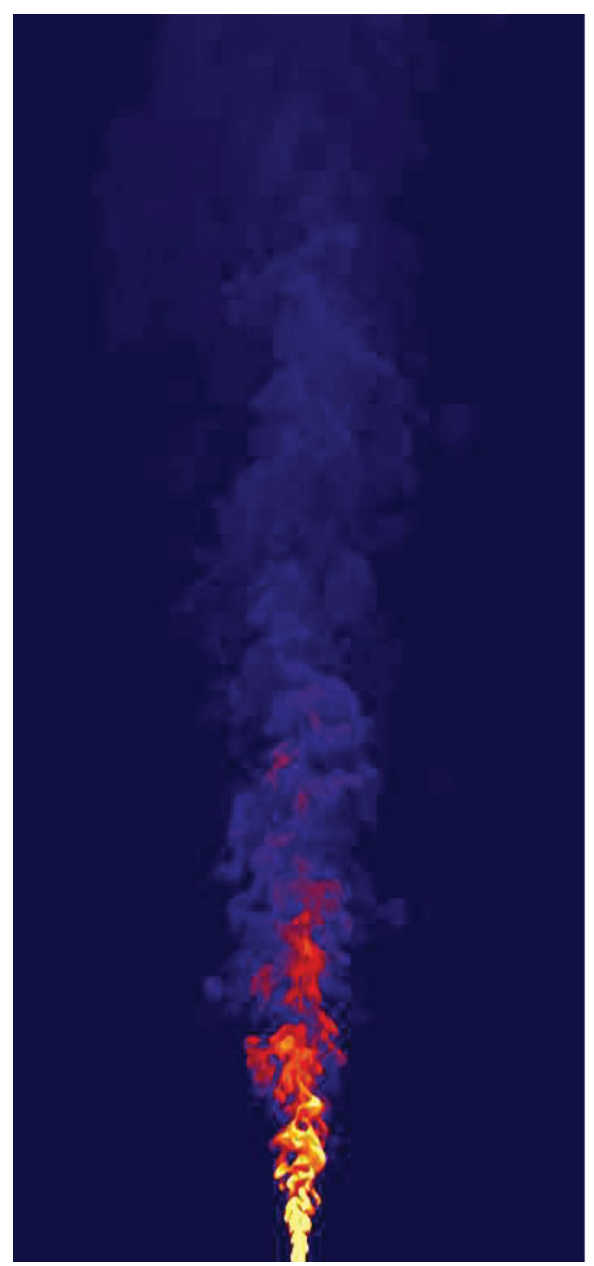

(b)

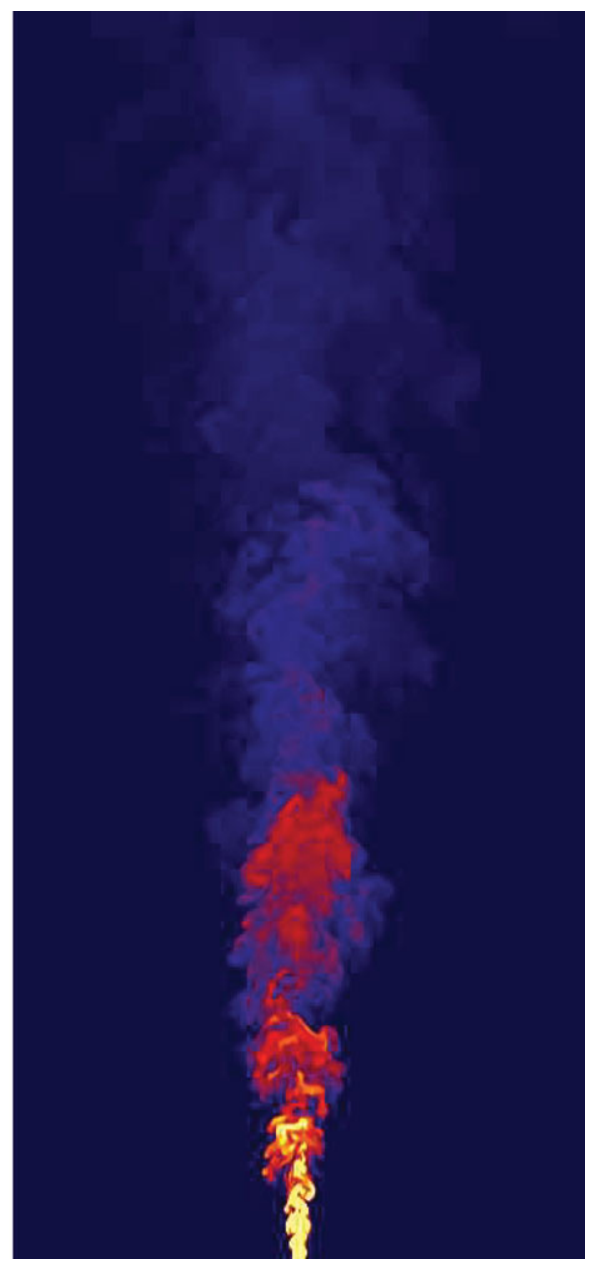

FIGURE 8. Instantaneous images of the temperature field. The field of view is the vertical plane $\left(y_{\star}=0 \mathrm{~m}\right)$ and the extent shown is $0.704 \mathrm{~m} \times 1.568 \mathrm{~m}$. The times shown are: $(a)$ before the source strength is increased; $(b) 15 \mathrm{~s}$ after the source strength is increased, the pulse is the red area at a height of approximately one third of the field of view above the source.

Instantaneous images from the numerical solutions are shown in figure 8 . The field shown is the temperature field and the plane shown is the central, $y_{\star}=0 \mathrm{~m}$, plane with horizontal and vertical extent $0.704 \mathrm{~m}$ and $1.568 \mathrm{~m}$, respectively. Figure $8(a)$ is the temperature field before the source strength is increased. It can be seen that the plume takes the expected slender form and the temperature decreases with distance from the source. Figure $8(b)$ is the temperature field $15 \mathrm{~s}$ after the source strength is increased. Again the plume has the slender form of figure $8(a)$, but it can now be seen that although the temperature initially decreases with distance from the source, there is an area, at a height of approximately one third of the field of view above the source, where the temperature increases. This area is the pulse and is hotter than the plume fluid ahead of it and behind it.

Figure 9 is a comparison between the predicted form of the pulse from $\S 2$, using the time-dependent plume equations (thin solid line), and the form found from the IAMR 


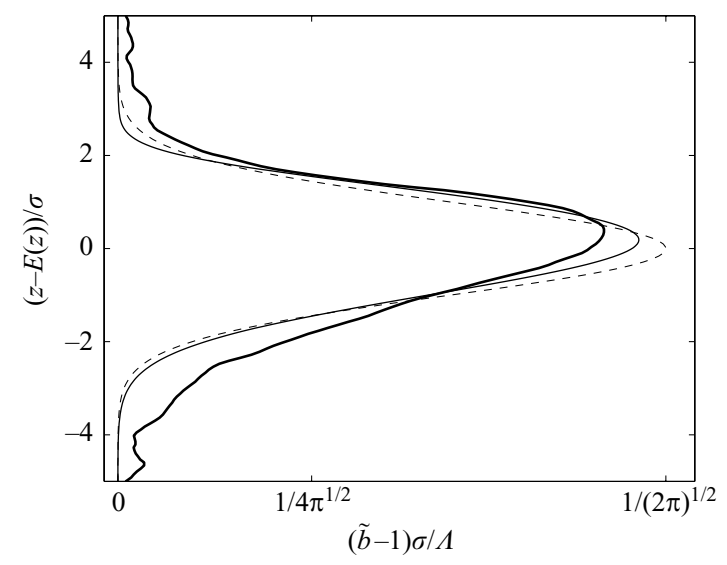

Figure 9. A comparison of the predicted pulse shape from the time-dependent plume equations in $\S 2$ (thin solid line) to the IAMR numerical simulation (thick solid line). The dashed line is a reference Gaussian.

simulations (thick solid line). A reference Gaussian is also shown (dashed). It can be seen that the time-dependent plume equations well predict the observed negatively skewed Gaussian form of the pulse, although the IAMR realizations indicate more area in the lower tail. This is not unexpected since the simulations model the vertical extent of the eddies within the plume, unlike the time-dependent plume equations. As observed in Scase et al. (2008), the vertical extent of the eddies means that some vertical homogenization occurs due to their overturning and mixing.

In figure $10(a)$ the evolution of the pulse rise height is plotted against time. The thin solid line is a best fit $t^{3 / 4}$ line, the dashed line is a best-fit power-law line and the thick solid line is from (2.9). It can be seen that there is an excellent agreement between the two best fit lines and that the $z_{h} \sim t^{3 / 4}$ prediction from both dimensional arguments and $\S 2$ works well. The data points generated by IAMR are seen to be far enough from the source to be well within the point source scaling regime, rather than the near distributed source scaling regime. Since the data points lie below the thick solid line, the height of the pulse is marginally over predicted.

Figure 10(b) shows the evolution of the pulse's volume against time. The thin solid line is a best fit $t^{9 / 4}$ line, the dashed line is a best-fit power-law line and the thick solid line is from (2.10). The agreement between the two best fit lines is good, but the $t^{9 / 4}$ prediction is slightly above the observed best fit power-law evolution of $t^{2.002}$. As the data points lie above the thick solid line, the volume of the pulse is under predicted, however this is consistent with the slight over prediction of the height pulse. Since the pulse is not moving as rapidly as predicted, more plume fluid enters the pulse from below than predicted.

The greatest discrepancy between the predictions of $\S 2$ and the IAMR simulations lies in the expected vertical extent of the pulse, shown in figure $10(c)$. The solid line is a best fit $t^{1 / 4}$ line and the dashed line is a best-fit power-law line. The model predictions of $\S 2$ are clearly inconsistent with the numerical simulations. The bestfit power law indicates a behaviour much closer to $\sigma \sim t^{1 / 2}$ (in fact the fit shown is $\sigma \sim t^{0.56}$ ). Again, we appeal to the missing physics in the time-dependent plume equations, the lack of any eddy structure, to explain this discrepancy. However, in the horizontal direction figure $10(d)$ indicates that the predicted linear $t$ scaling for 
(a)

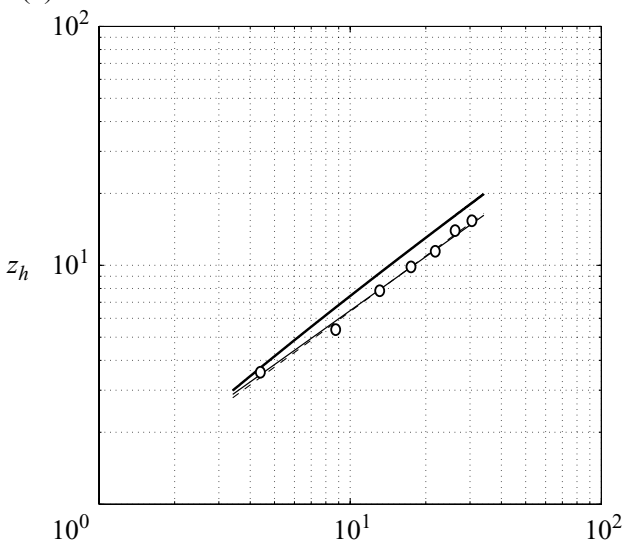

(c)

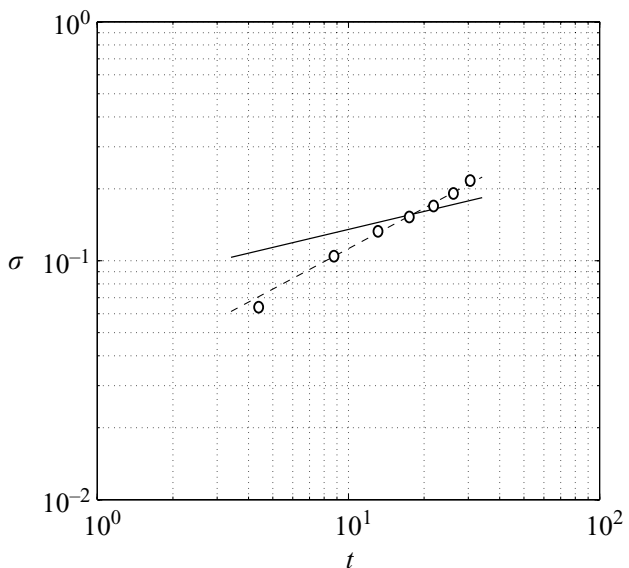

(b)

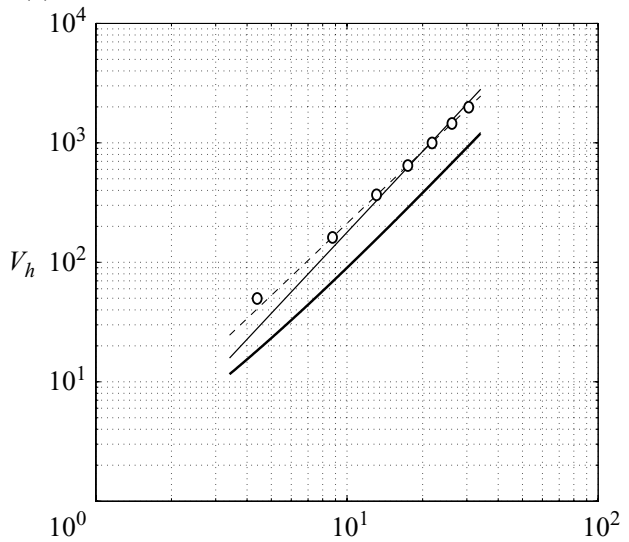

(d)

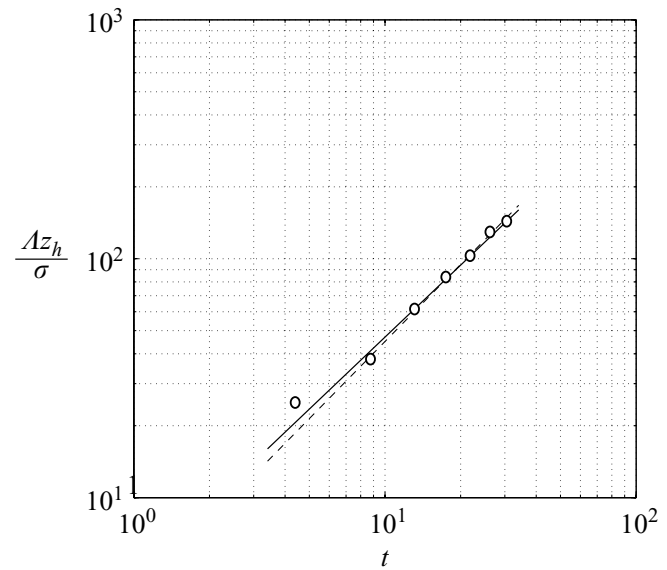

FIGURE 10. Plots comparing the IAMR simulations with the power-law predictions of $\S 2$. The thin solid lines are the best fits for the expected power-law behaviour and the dashed lines are best fit power-law lines. (a) The evolution of the pulse height $z_{h}$ against time $t$ with an expected $z_{h} \sim t^{3 / 4}$ behaviour. The thick solid line is from (2.9). The best-fit power law is $t^{0.772}$. (b) The evolution of the pulse volume $V_{h}$ against time $t$ with an expected $V_{h} \sim t^{9 / 4}$ behaviour. The thick solid line is from (2.10). The best-fit power law is $t^{2.002}$. (c) The evolution of the pulse's vertical extent $\sigma$ against time $t$ with an expected $\sigma \sim t^{1 / 4}$ behaviour. In fact the behaviour is much closer to $\sigma \sim t^{1 / 2}$ as the best-fit power law is $t^{0.562}$. $(d)$ The evolution of the pulse's horizontal extent $\Lambda z_{h} / \sigma$ against time $t$ with an expected linear $\Lambda z_{h} / \sigma \sim t$ behaviour. The best-fit power law is $t^{1.071}$.

$\Lambda z_{h} / \sigma$ is indeed reasonable. It is possible that a similar inconsistency in $\Lambda$ cancels the inconsistency in $\sigma$ favourably, leading to reasonable predictions for horizontal extent.

Figure 2 indicated the envelope of the pulses' evolution. Finally in this section we compare the evolution of this envelope for all the buoyancy flux increases considered in $\S 2$ (both point sources and distributed sources) and the numerical simulations in $\S 3$. As indicated in figure 2 , for a point source plume subjected to an increase in its source strength the rising pulse creates an envelope of initially zero horizontal extent that spreads faster than the conical steady plume profile. From the observed power laws in $\S 2$ it follows that there is no single power-law behaviour for the pulse envelope. It comprises both a $z$ contribution from the conical spreading, and 


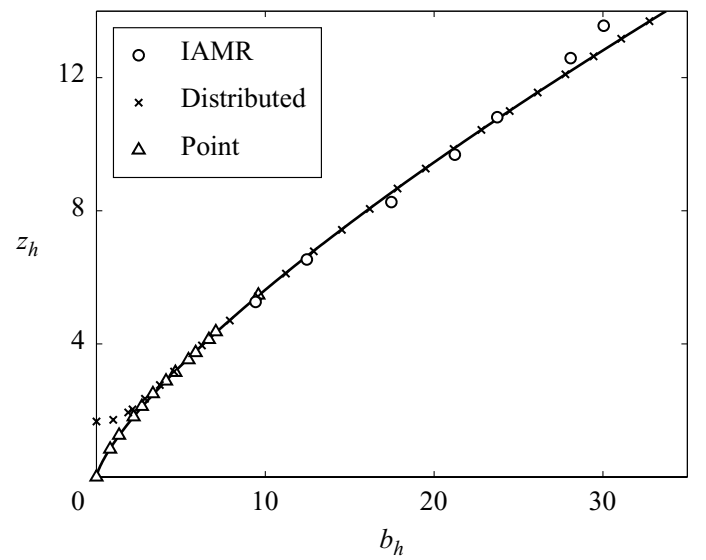

FIGURE 11. The pulse envelope. The origin of the distributed source data points and the IAMR data points are offset by a virtual origin correction. Far from the source the envelope is well modelled by a $z^{4 / 3}$ power law.

a $z^{4 / 3}$ contribution from the pulse's horizontal extent. However, if the horizontal extent of the pulse dominates the slender conical spreading, the envelope will be well approximated by a $z^{4 / 3}$ power law. Indeed, the triangular data points in figure 11 indicate that $z^{4 / 3}$ is a reasonable scaling for the pulse envelope for a point source plume subjected to a buoyancy increase (over seven orders of magnitude of buoyancy increase). The point source data has been scaled in the horizontal direction to sit on the $b_{h}=z^{4 / 3}$ line. The initial spreading of the pulse for a distributed source plume (cross data points in figure 11) is more rapid than its point source equivalent. The scalings in $\S 2$ indicate that if the pulse width dominates the steady conical plume width then $b_{h} \sim\left(z+z_{v s}\right)^{1 / 2}$ initially. In fact the calculated solutions indicate even more rapid growth with $b_{h} \sim\left(z+z_{v s}\right)^{0.4}$ near the distributed source. However, we observe that far from the source the evolution is governed by the point source power laws and $b_{h} \sim\left(z+z_{v s}\right)^{4 / 3}$ is a good approximation. The distributed source data points and IAMR data points have been non-dimensionalized and scaled in the horizontal direction. The result that $z_{h} \sim t^{3 / 4}$ and $b_{h} \sim t^{4 / 3}$ far from the source means that $b_{h}$ increases approximately linearly in time far from the source.

\section{Starting plumes}

\subsection{Introduction}

We consider the same set of seven solutions of the time-dependent plume equations as considered in $\S 2.2$ but in particular we investigate the limiting behaviour of the rise height, $z_{h}$ as $F_{0} \rightarrow 0$, with $F_{1}=1$. This behaviour is analogous to the starting plume problem. In order to verify that the rise height of an infinitesimal pulse, generated by $F_{0}=F_{1}-0$, is given by $(2.3)$ we also include the numerical solution with $F_{0}=0.9999$, $F_{1}=1$. For all eight cases we are interested in the coefficient $\lambda$ where

$$
z_{h \star}\left(t_{\star}\right)=\lambda\left(\frac{10}{9 \alpha^{(P)}}\right)^{1 / 2}\left(\frac{F_{1 \star}}{\pi \rho_{\infty \star \star}}\right)^{1 / 4} t_{\star}^{3 / 4} .
$$

The numerical results for $\lambda$ are given in table 1 . The relationship between $\lambda^{\prime}$ and $\lambda$ is given by $\lambda^{\prime}=(20 / 9)^{1 / 2} \lambda$. Turner (1962) found the vertical velocity of a starting-plume 
rises at approximately 0.61 times the vertical velocity of an established plume at the same height, i.e. $\lambda=0.61$.

\subsection{The top-hat starting plume model}

The starting plume model of Turner (1962) is a combination of the steady plume theory of Morton et al. (1956) and the buoyant vortex ring theory of Turner (1957). The key idea is that the lower section of the starting plume behaves as a 'steady plume' and this lower section feeds the front of the starting plume. The front of the starting plume is modelled as a thermal, which can itself be thought of as a buoyant vortex ring (Turner 1957). In particular, Levine (1959) modelled thermals as Hills' spherical vortex rings and this model was adopted by Turner (1962) for the starting-plume model.

Hill's (1894) spherical vortex solution of the Euler equations with radius $a_{\star}$, volume $V_{\star}$ and mean propagation velocity $W_{\star}$ has impulse $I_{\star}$ and circulation $\Gamma_{\star}$ given by

$$
\boldsymbol{I}_{\star}=\frac{\rho_{\star}}{2} \iiint_{V_{\star}} \boldsymbol{x}_{\star} \times \boldsymbol{\omega}_{\star} \mathrm{d} \boldsymbol{x}_{\star}=\left(0,0,2 \pi \rho_{\star} W_{\star} a_{\star}^{3}\right), \quad \Gamma_{\star}=\oint_{\gamma} \boldsymbol{u}_{\star} \cdot \mathrm{d} \boldsymbol{x}_{\star}=5 W_{\star} a_{\star} \cdot \quad(4.2 a, b)
$$

where $\gamma$ is a contour around the vortex ring core.

A buoyant vortex ring does not conserve its impulse $\boldsymbol{I}_{\star}=\left(0,0, I_{\star}\right)$ due to a buoyancy force $\boldsymbol{B}_{\star}=\left(0,0, B_{\star}\right)$, acting because the density of the vortex ring is lower than the density of the surrounding ambient fluid. Taking the acceleration due to gravity to be $g_{\star}$, the ambient fluid density to be $\rho_{\infty \propto \star}$ and the density of the thermal to be $\rho_{\star}^{(T)}$ then

$$
\frac{\mathrm{d} I_{\star}}{\mathrm{d} t_{\star}}=B_{\star}=g_{\star} V_{\star}\left(\rho_{\circ \star \star}-\rho_{\star}^{(T)}\right) .
$$

As noted in $\S 1.2$, throughout this section a superscript $\mathrm{T}$ denotes quantities for thermals. Even though $\rho_{\star}^{(T)}$ and $V_{\star}$ change in time, the total buoyancy force $\boldsymbol{B}_{\star}$ remains constant since no buoyant fluid is supplied or removed during the flow.

Figure 12 shows the schematic set-up for a starting plume in a top-hat framework. We choose the top-hat framework for illustrative purposes, and for consistency with the time-dependent plume model in Scase et al. (2006b). A steady plume, of the form considered by Morton et al. (1956) extends from a point source at $z_{\star}=0$ to the base of the thermal cap at $z_{\star}=z_{h \star}$ where it has radius $b_{h \star}$. The thermal cap is assumed to be spherical and has radius $a_{\star}$. The centre of the thermal cap is at $z_{\star}=z_{c \star}$ and rises with velocity $w_{\star}=w_{c \star}$. The base of the thermal cap is located at $z_{\star}=z_{h \star}$ and rises with velocity $w_{\star}=w_{h \star}^{(T)}$. The flow is similar at all times (Turner 1973) and so we define the ratio

$$
\frac{a_{\star}}{z_{c \star}}=\alpha^{(T)},
$$

a constant. It follows that $\mathrm{d} a_{\star} / \mathrm{d} t_{\star}=\alpha^{(T)} w_{c \star}$ which governs the entrainment into the thermal cap. This relation shows that the entrainment into the thermal cap is proportional to the vertical velocity at the centre of the thermal cap, similarly to the standard entrainment assumption for a plume. It follows from the geometrical relation $z_{c \star}=z_{h \star}+a_{\star}$ (see figure 12) that

$$
w_{h \star}^{(T)}=\left(1-\alpha^{(T)}\right) w_{c \star} .
$$

The plume's vertical velocity at $z_{\star}=z_{h \star}$ is given by $w_{\star}=w_{h \star}^{(P)}$ and is greater than vertical velocity of the base of the thermal cap at the same height, i.e. $w_{h_{\star}}^{(P)}>w_{h \star}^{(T)}$.

For the plume in the top-hat framework the mass flux $Q_{\star}$, momentum flux $M_{\star}$ and buoyancy flux $F_{\star}$ are as defined in (1.2). The steady plume equations of Morton $e t$ al. 


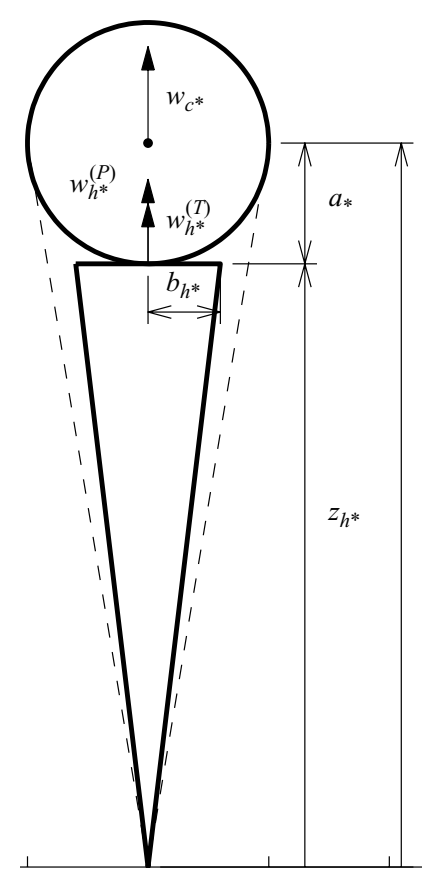

FIGURE 12. Schematic set-up of the top-hat equivalent of Turner's (1962) starting-plume model. The top of the plume is at $z_{\star}=z_{h \star}$ and the thermal is of radius $a_{\star}$ centred at $z_{\star}=z_{c \star}$. The vertical velocity in the plume at $z_{\star}=z_{h \star}$ is $w_{\star}=w_{h \star}^{(P)}$ and is greater than the vertical velocity in the thermal at the same height, $w_{\star}=w_{h \star}^{(T)}$. The velocity of the centre of the thermal is $w_{\star}=w_{c \star}$.

(1956) corresponding to (1.3) are

$$
\frac{\mathrm{d} Q_{\star}}{\mathrm{d} z_{\star}}=2 \alpha^{(P)}\left(\pi \rho_{\infty \star}\right)^{1 / 2} M_{\star}^{1 / 2}, \quad \frac{\mathrm{d} M_{\star}}{\mathrm{d} z_{\star}}=\frac{Q_{\star} F_{\star}}{M_{\star}}, \quad \frac{\mathrm{d} F_{\star}}{\mathrm{d} z_{\star}}=0 .
$$

The vertical velocity and the plume radius at the top of the plume at height $z_{\star}=z_{h \star}$ are then given, respectively, by

$$
w_{h \star}^{(P)}=\frac{5}{6 \alpha^{(P)}}\left(\frac{9 \alpha^{(P)}}{10}\right)^{1 / 3}\left(\frac{F_{0 \star}}{\pi \rho_{\infty \star}}\right)^{1 / 3} z_{h \star}^{-1 / 3}, \quad b_{h \star}=\frac{6 \alpha^{(P)}}{5} z_{h \star} .
$$

The surface $z_{\star}=z_{h \star}$ rises with vertical velocity $w_{h \star}^{(T)}$ and so because $w_{h \star}^{(P)}>w_{h \star}^{(T)}$ the plume supplies mass, momentum and buoyancy to the thermal. Denoting the mass, momentum and buoyancy fluxes from the plume at $z_{\star}=z_{h_{\star}}$ into the thermal at $z_{\star}=z_{h \star}$ by $\hat{Q}_{\star}, \hat{M}_{\star}$ and $\hat{F}_{\star}$, respectively, it follows that

$$
\hat{Q}_{\star}=\int_{0}^{2 \pi} \int_{0}^{b_{\star}} \rho_{\star}^{(P)}\left(w_{h \star}^{(P)}-w_{h \star}^{(T)}\right) r_{\star} \mathrm{d} r_{\star} \mathrm{d} \theta=Q_{\star}\left(z_{h \star}\right)\left[1-\frac{w_{h \star}^{(T)}}{w_{h \star}^{(P)}}\right],
$$

and similarly

$$
\hat{M}_{\star}=M_{\star}\left(z_{h \star}\right)\left[1-\frac{w_{h \star}^{(T)}}{w_{h \star}^{(P)}}\right], \quad \hat{F}_{\star}=F_{\star}\left(z_{h \star}\right)\left[1-\frac{w_{h \star}^{(T)}}{w_{h \star}^{(P)}}\right] .
$$

Unlike the isolated thermal, the buoyancy force on the starting-plume thermal cannot be considered constant due to the supply of buoyancy from the plume below, i.e. 
since $\hat{F}_{\star} \neq 0$. The buoyancy force acting on the starting-plume thermal obeys

$$
\frac{\mathrm{d} B_{\star}}{\mathrm{d} t_{\star}}=\hat{F}_{\star} .
$$

The rate of change with time of the impulse of the starting-plume thermal can also no longer be considered constant due to the supply of momentum from the plume. Hence

$$
\frac{\mathrm{d} I_{\star}}{\mathrm{d} t_{\star}}=B_{\star}+\hat{M}_{\star} .
$$

Following Turner (1962) we appeal to similarity to write $w_{h \star}^{(T)}=c_{1} w_{h \star}^{(P)}$. It follows therefore from $(4.7 a)$ that

$$
z_{h \star}=\left(\frac{10}{9 \alpha^{(P)}}\right)^{1 / 2}\left(\frac{F_{0 \star}}{\pi \rho_{\infty \star \star}}\right)^{1 / 4}\left(c_{1} t_{\star}\right)^{3 / 4},
$$

and hence

$$
M_{\star}\left(z_{h \star}\right)=F_{0 \star} c_{1} t_{\star} .
$$

It follows from $(4.8 b, c),(4.9),(4.10),(4.11)$ and the conditions $B_{\star}(0)=0 \mathrm{~kg} \mathrm{~m} \mathrm{~s}^{-2}$, $I_{\star}(0)=0 \mathrm{~kg} \mathrm{~m} \mathrm{~s}^{-1}$ that

$$
B_{\star}=F_{0 \star}\left(1-c_{1}\right) t_{\star}, \quad I_{\star}=F_{0 \star}\left(1-c_{1}^{2}\right) \frac{t_{\star}^{2}}{2} .
$$

Finally, based on the observation that the impulse behaves similarly to the Hill's spherical vortex ring model described above (4.2) we write $I_{\star} \propto \pi \rho_{\infty} w_{c \star} a_{\star}^{3}$ and therefore for some constant $c$

$$
I_{\star}=\frac{\pi \rho_{\infty{ }_{\infty}}}{c} w_{c \star} a_{\star}^{3}=\frac{\pi \rho_{\infty{ }_{\star}} c_{1}}{c\left(1-\alpha^{(T)}\right)} w_{h \star}^{(P)} a_{\star}^{3},
$$

where we have applied the similarity assumption and (4.5) to write $w_{c \star}$ in terms of $w_{h_{\star}}^{(P)}$. The expression for the impulse in (4.14) is equated to that given in $(4.13 \mathrm{~b})$ and $t_{\star}$ is substituted for by integrating the right-hand side of $(4.7 a)$ multiplied by $c_{1}$ (since the required vertical velocity is that of the base of the thermal, not that of the plume velocity) yielding

$$
a_{\star}^{3}=\frac{5}{16} \frac{c\left(1-c_{1}^{2}\right)\left(1-\alpha^{(T)}\right)}{c_{1}^{3} \alpha^{(P)}} b_{h \star}^{3} .
$$

As $b_{h \star}$ is linearly dependent on $z_{\star}(4.7 b)$ the thermal cap radius $a_{\star}$ is also linearly dependent on $z_{\star}$. We now make the final observation that since $a_{\star} / z_{c \star}=\alpha^{(T)}$ we have then

$$
a_{\star}=\frac{5}{6 \alpha^{(P)}} \frac{\alpha^{(T)}}{1-\alpha^{(T)}} b_{h \star} .
$$

The relations in (4.15) and (4.16) demonstrate the internal consistency of Turner's (1962) model.

The mass flux into the thermal cap from the ambient fluid, not including the mass flux from the plume into the thermal, is denoted as $Q_{\star}^{(E)}$. The total mass flux into the thermal cap, including entrained ambient fluid as well as buoyant fluid supplied by the plume, is denoted by $Q_{\star}^{(T)}$. Hence

$$
Q_{\star}^{(T)}=Q_{\star}^{(E)}+\hat{Q}_{\star}
$$


Since the thermal radius $a_{\star}$ obeys (4.16) it follows that

$$
Q_{\star}^{(T)}=\frac{\mathrm{d}}{\mathrm{d} t_{\star}}\left(\rho_{\infty \star \star} \frac{4 \pi}{3} a_{\star}^{3}\right)=\rho_{\infty \star} 4 \pi a_{\star}^{2} \alpha^{(T)} w_{c \star} .
$$

Hence the ratio of plume fluid entering the cap at any instant to the total entrainment into the thermal cap at any instant is given by

$$
\frac{\hat{Q}_{\star}}{Q_{\star}^{(T)}}=\left(\frac{6 \alpha^{(P)}}{5}\right)^{2} \frac{1-c_{1}}{4 c_{1}}\left(\frac{1-\alpha^{(T)}}{\alpha^{(T)}}\right)^{3} .
$$

Turner (1962) stated that 'about half the fluid entering the spherical cap at any instant comes from below'. However, a small typographical error in (18) in his paper whereby, in his notation, the factor $\beta$ should be replaced by $(R / b)^{2}$, leads to this ratio changing from 0.49 to 0.40 .

We make the assumption that the exact form of profile chosen for a given plume model, e.g. top-hat or Gaussian etc. has no effect on either the ratio $w_{h \star}^{(T)} / w_{h \star}^{(P)}$ or $\hat{Q}_{\star} / Q_{\star}^{(T)}$. As is usual we choose $\alpha^{(P)}=0.10$ for a top-hat plume and choose $\alpha^{(T)}$ such that $\hat{Q}_{\star} / Q_{\star}^{(T)}=0.40$ which leads to $\alpha^{(T)}=0.20$. It should be noted then that the ratio of $\alpha^{(P)}$ for a Gaussian plume compared to a top-hat plume $(0.09 / 0.10)$ is then also equal to the ratio of $\alpha^{(P)}$ for the Gaussian thermal cap compared to the top-hat thermal cap $(0.18 / 0.20)$.

Finally we observe that our time-dependent model, which lacks both the modelling of the dynamics in the thermal cap and the extra entrainment of ambient fluid through the surface of the thermal cap, will therefore under-entrain by the ratio

$$
\frac{Q_{\star}^{(E)}}{Q_{\star}^{(T)}+Q_{\star}\left(z_{h_{\star}}\right)}=1-\frac{2-c_{1}}{1+4 c_{1}\left(\frac{\alpha^{(T)}}{1-\alpha^{(T)}}\right)^{3}\left(\frac{5}{6 \alpha^{(P)}}\right)^{2}}=0.36 .
$$

\subsection{Comparison with Turner (1962)}

If $F_{0}=F_{1}-0$ then the rise height of the infinitesimal 'pulse' is given by the steady Morton et al. (1956) model (2.3). We wish to compare this to the situation with $F_{0}=0$ and $F_{1}=1$, the starting plume set up. As in $\S 2.2$, we write

$$
z_{h}=\lambda\left(\frac{20}{9}\right)^{1 / 2} t^{k}
$$

the results of numerical simulations are given in table 1 and shown in figure 13. Figure 13(a) shows the evolution of a 'starting plume' as modelled by the time-dependent plume equations (1.3). The position of the top of the starting plume was found by defining a passive tracer concentration field (see Scase et al. 2008, (3.3)) that was initially zero. At $t=0$ the concentration at the source was increased to 1 and the extent of the starting plume was taken to be all heights for which the concentration was above the numerical noise level of $10^{-8}$. The image shows the plume establishing the usual steady $b=3 z / 5$ profile, but its head shape is flatter and wider than is observed in starting plumes. As before though, top-hat estimates of horizontal extent need not be the true horizontal extent of a real plume, as discussed in $\S 2.2$.

Figure 13(b) is a plot of the calculated value of Turner's (1962) factor, $\lambda$, for each of the buoyancy increases considered. The plot demonstrates that, qualitatively, if the initial plume strength is weak then the pulse propagates up the plume more slowly than if the initial plume strength is strong. Using the differences between $k$ and $3 / 4$ as an estimate, the errors are of order $2 \%$ (see table 1). Keeping a fixed 
(a)

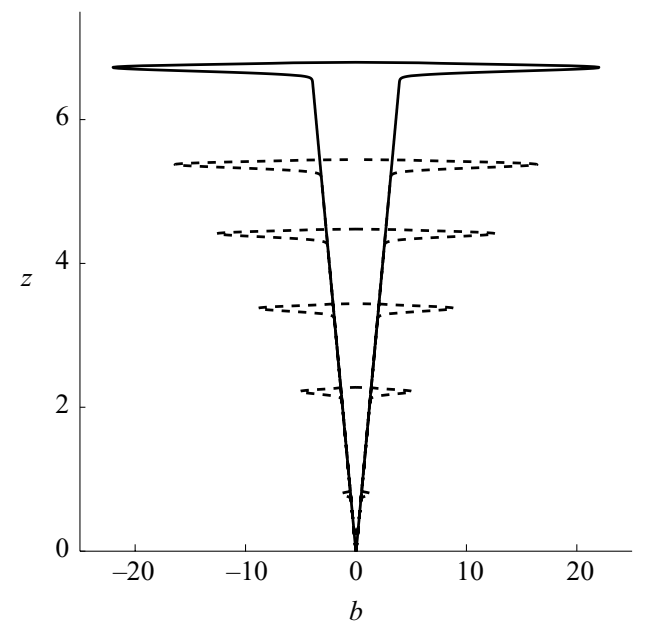

(b)

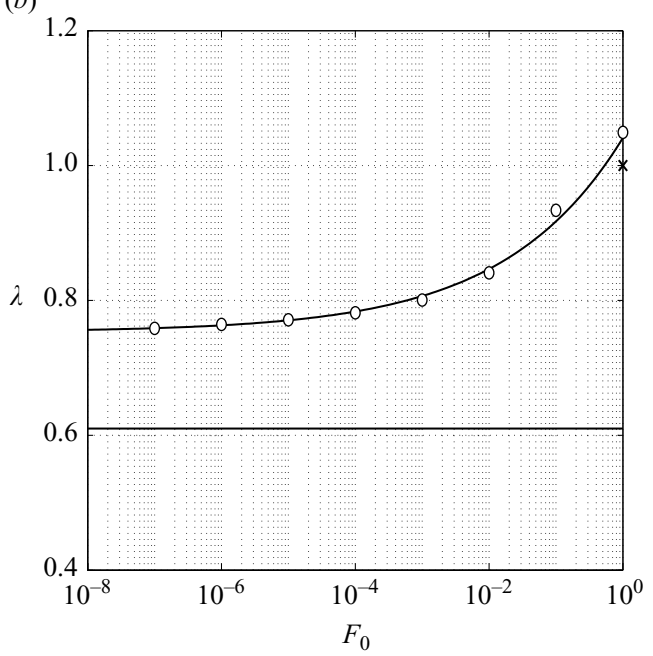

Figure 13. (a) The evolution of the approximation to a starting plume from the time-dependent plume equations (1.3). The times shown are $t=0.56,2.16,3.76,5.36,6.96$ (dashed) and $t=8.56$ (solid). (b) Graph showing the limiting behaviour of $\lambda$ as $F_{1}=1$ and $F_{0} \rightarrow 0$. The horizontal line is Turner's empirical value of $\lambda=0.61$. The cross is the theoretical value based on the steady Morton et al. (1956) solution. The solid curved line is a best fit through the data points, predicting a minimum value of $\lambda$ as $F_{0} \rightarrow 0$ of $\lambda=0.75$.

final non-dimensional buoyancy flux, $F_{1}=1$, the initial non-dimensional buoyancy flux, $F_{0}$, was reduced from 0.9999 to a value of $10^{-7}$. When $F_{0}=0.9999$ the value of $\lambda$ and $k$ are in good agreement with the steady Morton et al. (1956) model where $F_{0}=F_{1}=1$, i.e. $\lambda=1$ and $k=3 / 4$. As $F_{0}$ is progressively reduced, the value of $k$ remains approximately constant at $k \approx 3 / 4$. The factor $\lambda$ reduces and tends to a constant value $\approx 0.75$. Approximately therefore, we find based on the time-dependent plume model

$$
z_{h \star}=0.75 \times\left(\frac{10}{9 \alpha^{(P)}}\right)^{1 / 2}\left(\frac{F_{0 \star}}{\pi \rho_{\infty \star \star}}\right)^{1 / 4} t_{\star}^{3 / 4} .
$$

There is therefore a discrepancy between Turner's factor, $\lambda=0.61$ and the present value $\lambda=0.75$, a difference of $23 \%$.

We now use (4.20) to compare the prediction given in (4.22) to the known riseheight velocity given in (2.3) by replacing $\alpha^{(P)}$ with $1.36 \alpha^{(P)}$ (accounting simply for the extra entrainment through the cap) and find

$$
z_{h \star}=0.65 \times\left(\frac{10}{9 \alpha^{(P)}}\right)^{1 / 2}\left(\frac{F_{0 \star}}{\pi \rho_{0 \star \star}}\right)^{1 / 4} t_{\star}^{3 / 4} .
$$

The factor $\lambda=0.65$ compares well with Turner's (1962) factor $\lambda=0.61 \pm 0.05$ given that no attempt to model the dynamics of the thermal cap has been made. This suggests that the main effect of the thermal cap is to entrain ambient fluid with negligible vertical velocity. This extra entrained mass acts as a drag on the starting plume which slows its vertical propagation compared to that of an established steady plume. It would appear that the precise nature of the internal dynamics of the thermal cap is not significant in determining the rise height. 


\section{Conclusions}

The time-dependent generalization of the successful plume model (Morton et al. 1956) derived in Scase et al. (2006b) is able to model the evolution of a turbulent plume that is subjected to changes in its source strength, both decreases and increases. It has been shown previously in the literature that a plume that is subjected to a rapid decrease in its source strength forms a narrow region well modelled by the power-law similarity solution to the governing equations. No such power-law solution to the equations exists for a plume subjected to a rapid increase in source strength. We have investigated two buoyancy increase scenarios of practical interest, the effect of rapid buoyancy increase on an established plume and the 'startingplume'.

In the first of these scenarios it was shown that by increasing the source strength of an established plume, a pulse travels up the plume. It was shown that for a point source plume, and a pure distributed source plume far from the source, that the pulse propagates upwards with a $t^{3 / 4}$ scaling. This is the same scaling with time as for a passive tracer propagating vertically within a classical steady plume. This $t^{3 / 4}$ scaling may have been expected for moderate increases in buoyancy flux given the established steady plume model. It was shown however that this scaling applies across a large number of orders of magnitude of buoyancy increase where the inertia of the slow plume moving fluid ahead of the pulse becomes important. It was also shown that near the source of a distributed source plume, the initial momentum of the plume fluid dominates the propagation velocity of the pulse and it initially propagates linearly in time. The volume of extra plume fluid contained within the pulse was shown to follow a $t^{9 / 4}$ scaling for a point source plume or a distributed source plume far from the source. This scaling might have been predicted given the $t^{3 / 4}$ behaviour of the pulse's rise height. Near to the source of the distributed source plume the volume of the pulse was shown to be dominated by the initial mass flux of the plume and hence followed a linear scaling.

The implicit LES approach employed in $\S 3$ is well suited to high-Reynolds-number shear flows. For a sufficiently large Reynolds number turbulent plumes and jets become, to a good approximation, Reynolds number independent. This independence is due to the dominance of the Reynolds stresses compared to the viscous stresses. The details not captured explicitly by the ILES approach therefore do not play a significant role in the dynamics. For a fuller discussion of ILES methods applied to high-Reynolds-number shear flows see Oran \& Boris (1993) and Fureby \& Grinstein (1999).

The behaviours of the pulse's rise height and volume with time are the most important physical results, giving, for example, time scales for evolution and accumulation of concentrations of pollutants in the atmosphere. However, it was also shown that the pulse remains self-similar across a large number of orders of magnitude of buoyancy increase, and persists in self-similar fashion for long times. Furthermore the pulse remained self-similar across a number of orders of magnitude of source size. This observation offers encouragement in terms of the possibility of finding a universal solution.

A starting plume is fundamentally different to an established plume that has its source strength varied. At the top of a starting plume is a 'thermal cap' with a vortex-ring-like structure that has internal dynamics similar to those of a vertically propagating vortex ring, and furthermore the plume is entraining ambient fluid through this thermal cap. The horizontally averaged time-dependent plume equations cannot account for either of these physical processes. However it is still of interest 
to see how well the model performs in comparison to the accepted starting plume model with a view to unifying the classical steady plume model of Morton et al. (1956) and the starting plume model of Turner (1962). It would be preferable to have one universal model for plume dynamics rather than requiring a specific model for each different scenario. It was shown that despite the inability of the time-dependent plume equations to model all the physics of a starting plume it still predicted the rise height of a starting plume reasonably (over predicting the rise height by $23 \%$ ). The main reason for the over prediction was the lack of entrainment of ambient fluid, with zero vertical momentum, through a thermal cap. If the entrainment coefficient was artificially increased to account for the extra entrainment of ambient fluid, with zero vertical inertia, the predicted rise heights matched extremely closely.

The authors are greatly indebted to Dr S. B. Dalziel and Professor A. W. Woods for many useful discussions. M. M. Scase gratefully acknowledges funding from the US-UK Fulbright Commission. C. P. Caulfield gratefully acknowledges the support of the 2008 Summer Study program in Geophysical Fluid Dynamics at Woods Hole Oceanographic Institution where the preparation of this manuscript took place.

\section{Note added in Proof}

A similar set of equations to (1.3) was posed for Gaussian profile plumes in Delichatsios (1979). The authors are grateful to Professor Vul'fson for drawing their attention to this paper.

\section{Appendix A. Two increases in the source buoyancy flux of a turbulent plume}

The non-dimensional time-dependent plume equations (Scase et al. 2008, $(2.4 a-c)$ ) can be written as

$$
\frac{\partial \boldsymbol{Q}}{\partial t}+\boldsymbol{A} \frac{\partial \boldsymbol{Q}}{\partial z}+\boldsymbol{h}=\mathbf{0},
$$

where $\boldsymbol{Q}=(Q, M, F)^{\top}$,

$$
\boldsymbol{A}=\left(\begin{array}{ccc}
0 & 1 & 0 \\
-\frac{M^{2}}{Q^{2}} & 2 \frac{M}{Q} & 0 \\
-\frac{F M}{Q^{2}} & \frac{F}{Q} & \frac{M}{Q}
\end{array}\right) \quad \text { and } \quad \boldsymbol{h}=\left(\begin{array}{c}
-\frac{Q F}{M} \\
\frac{M^{5 / 2}}{Q^{2}}-2 F \\
\frac{F M^{3 / 2}}{Q^{2}}-\frac{F^{2}}{M}
\end{array}\right)
$$

The matrix $\boldsymbol{A}$ has three real repeated eigenvalues and a set of three linearly independent left eigenvectors, $\boldsymbol{e}_{i}$, for $i=1, \ldots, 3$. We define a matrix $\boldsymbol{L}$ as a matrix of left eigenvectors such that

$$
\boldsymbol{L}=\left(\begin{array}{l}
\boldsymbol{e}_{1} \\
\boldsymbol{e}_{2} \\
\boldsymbol{e}_{3}
\end{array}\right)=\left(\begin{array}{rrr}
0 & F & -M \\
F & 0 & -Q \\
M & -Q & 0
\end{array}\right) \quad \text { satisfies } \quad \boldsymbol{L} \boldsymbol{A}=\frac{M}{Q} \boldsymbol{L} .
$$

Although intuition may lead us to expect the system to be hyperbolic, $L$ is singular, $\boldsymbol{A}$ cannot therefore be diagonalized and the system must be regarded as parabolic. An example of the parabolic nature of the solution space is shown in figure 14. The presence of the repeated eigenvalue, $M / Q$, forces wave information to travel through 
(a)

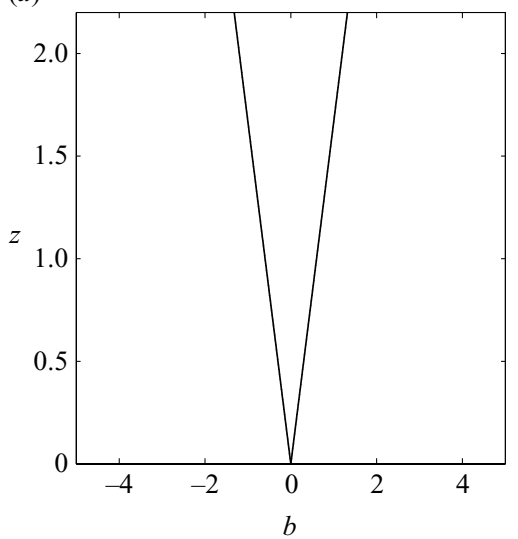

(c)

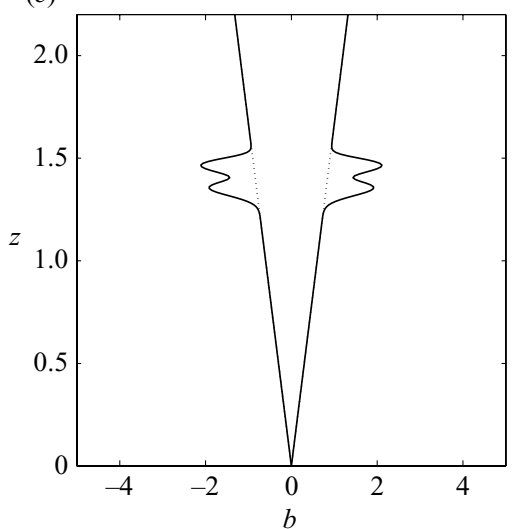

(b)

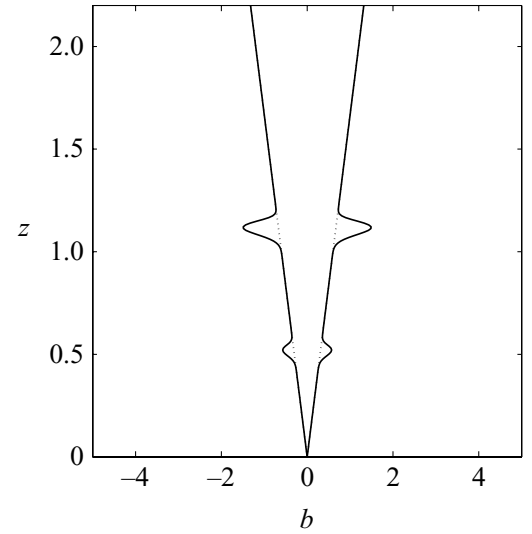

(d)

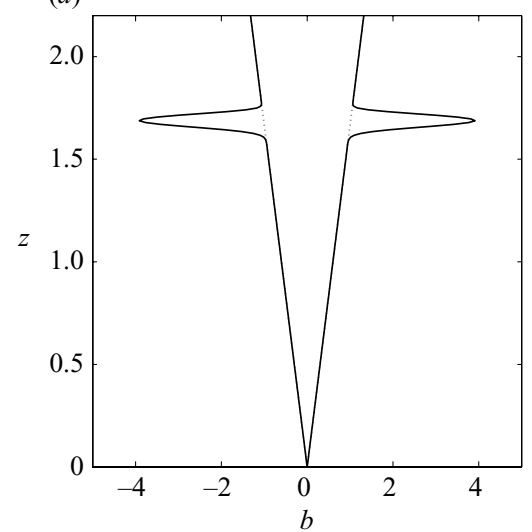

FIgURE 14. $(a-d)$ The evolution of two increases in the source buoyancy flux. The solid line is the plume radius, the dotted line is the steady Morton et al. (1956) plume radius. The buoyancy flux at the source is initially $F_{0}=1$. At $t=0.30$ the source strength is increased to $F_{0}=10$, and then at $t=1.04$ the source strength is increased again to $F_{0}=100$. The images demonstrate that the second perturbation catches the first and coalesces with it. $(a) t=0,(b) t=1.18$, (c) $t=1.57$ and $(d) t=1.78$.

the system at the local velocity, i.e. waves cannot pass through each other. Figure 14 shows an initially steady plume that is subject to two increases in the buoyancy flux at the source, separated by time $t=0.74$. A slow-moving pulse is seen to propagate up the plume followed by a much faster moving pulse. The faster moving pulse catches the slower moving pulse, but does not pass through the slower moving pulse. Instead, both pulses coalesce and move together as one.

\section{Appendix B. Independence of numerical solutions to resolution}

Numerical solutions of the time-dependent plume model indicate that the estimates for the normalized shape, height and volume of the pulse are robust, as is the $t^{1 / 4}$ scaling for the vertical extent of the pulse, see table 3 . However, the coefficients for the scaling of the vertical and horizontal extent of the pulse are sensitive to resolution. With increasing spatial resolution, the pulse becomes ever smaller in vertical extent and larger in horizontal extent. Increasing the vertical resolution necessarily requires 


\begin{tabular}{|c|c|c|c|c|c|c|c|c|c|c|c|c|c|}
\hline \multirow[b]{2}{*}{$F_{0}$} & \multirow[b]{2}{*}{$F_{1}$} & \multicolumn{6}{|c|}{ Low resolution $n_{z}=250, n_{t}=5000$} & \multicolumn{6}{|c|}{ High resolution $n_{z} \geqslant 5000, n_{t}=250000$} \\
\hline & & $\lambda_{z}^{\prime}$ & $\kappa_{z}$ & $\lambda_{V}^{\prime}$ & $\kappa_{V}$ & $\lambda_{\sigma}^{\prime}$ & $\kappa_{\sigma}$ & $\lambda_{z}^{\prime}$ & $\kappa_{z}$ & $\lambda_{V}^{\prime}$ & $\kappa_{V}$ & $\lambda_{\sigma}^{\prime}$ & $\kappa_{\sigma}$ \\
\hline $10^{-1}$ & 1 & 1.334 & 0.762 & 0.803 & 2.199 & 0.074 & 0.228 & 1.392 & 0.755 & 0.698 & 2.228 & 0.010 & 0.245 \\
\hline $10^{-2}$ & 1 & 1.200 & 0.763 & 0.989 & 2.186 & 0.060 & 0.240 & 1.254 & 0.755 & 0.843 & 2.229 & 0.008 & 0.240 \\
\hline $10^{-3}$ & 1 & 1.136 & 0.764 & 1.078 & 2.173 & 0.054 & 0.244 & 1.193 & 0.756 & 0.897 & 2.227 & 0.007 & 0.240 \\
\hline $10^{-4}$ & 1 & 1.092 & 0.772 & 1.155 & 2.157 & 0.052 & 0.246 & 1.165 & 0.756 & 0.930 & 2.224 & 0.007 & 0.240 \\
\hline $10^{-5}$ & 1 & 1.074 & 0.770 & 1.237 & 2.138 & 0.051 & 0.246 & 1.150 & 0.758 & 0.957 & 2.220 & 0.007 & 0.240 \\
\hline $10^{-6}$ & 1 & 1.044 & 0.778 & 1.328 & 2.117 & 0.050 & 0.244 & 1.139 & 0.758 & 0.982 & 2.215 & 0.007 & 0.239 \\
\hline $10^{-7}$ & 1 & 1.013 & 0.787 & 1.429 & 2.096 & 0.050 & 0.241 & 1.131 & 0.760 & 1.010 & 2.209 & 0.007 & 0.240 \\
\hline
\end{tabular}


an increase in the temporal resolution for convergence and stability, and an increase in temporal resolution changes the boundary condition as the 'step change' in source conditions becomes apparently more rapid, promoting a more sharper solution. Due to the limitations in computational power it is not clear when full convergence will be attained. A rapid linear increase in source strength over a finite time, that is therefore independent of temporal resolution unlike the step change, has also been considered. Initial investigations indicate a small region above the source where yet another scaling regime exists. Above this region the plume adjusts and behaves as if a step change occurred at the source. A fuller discussion is beyond the scope of the present paper and as such only the robust results are discussed.

The solutions found in $\$ 2.2$ were calculated using the method described in Scase et al. (2006b, appendix A) except that in the present case solutions were stepped forward in time using a fourth-order Runge-Kutta scheme. This solution technique, whereby perturbations to the steady Morton et al. (1956) solutions are considered, is necessary to remove the numerical difficulties associated with infinite velocities at a point source. However this scheme is not suitable for the distributed source plumes discussed in $\S 2.3$. The solutions in $\S 2.3$ were found using a separate solver based on the formulation in (A 1) and (A 2). Using two separate solvers based on two different solution methods demonstrates the robustness of the results presented herein.

\section{REFERENCES}

Almgren, A. S., Bell, J. B., Colella, P., Howell, L. H. \& Welcome, M. L. 1998 A conservative adaptive projection method for the variable density incompressible Navier-Stokes equations. J. Comp. Phys. 142, 1-46.

Almgren, A. S., Bell, J. B. \& Crutchfield, W. Y. 2000 Approximate projection methods. Part I. Inviscid analysis. SIAM J. Sci. Comp. 22, 1139-1159.

Aspden, A. J., Nikiforakis, N., Dalziel, S. B. \& Bell, J. B. 2008 Analysis of implicit LeS methods. Comm. Appl. Math. Comput. Sci. 3, 103-126.

Bell, J. B., Colella, P. \& Howell, L. H. 1991 An efficient second-order projection method for viscous incompressible flow. In 10th A.I.A.A. Computational Fluid Dynamics Conference, Honolulu, US.

BoRIS, J. P. 1990 On large eddy simulation using subgrid turbulence models. Comment 1. In Lecture notes in Physics (ed. J. L. Lumley), vol. 357, pp. 344-353. Springer Verlag.

Boris, J. P., Grinstein, F. F., Oran, E. S. \& Kolbe, R. L. 1992 New insights into large eddy simulation. Fluid Dyn. Res. 10, 199-229.

CAulfield, C. P. 1991 Stratification and buoyancy in geophysical flows. PhD thesis, University of Cambridge, UK.

Caulfield, C. P. \& Woods, A. W. 1995 Plumes with non-monotonic mixing behaviour. Geophys. Astrophys. Fluid Dyn. 79, 173-199.

Colella, P. 1985 A direct Eulerian MUSCL scheme for gasdynamics. SIAM J. Sci. Stat. Comp. 6, 104-117.

Colella, P. 1990 A multidimensional second order Godunov scheme for conservation laws. J. Comp. Phys. 87, 171-200.

Delichatsios, M. A. 1979 Time similarity analysis of unsteady buoyant plumes in neutral surroundings. J. Fluid Mech. 93, 241-250.

Drikakis, D., Fuerby, C. Grinstein, F. F. \& Youngs, D. L. 2007 Simulation of transition and turbulence decay in the Taylor-Green vortex. J. Turbul. 8, 1-12.

Fureby, C. \& GRINSTEIN, F. F. 1999 Monotonically integrated large eddy simulations of free shear flows. AIAA J. 37, 544-556.

Grinstein, F. F., Margolin, L. G. \& Rider, W. J. 2007 Implicit Large Eddy Simulation. Cambridge University Press.

HiLl, M. J. M. 1894 On a spherical vortex. Phil. Trans. R. Soc. A 185, 213-245. 
Hunt, G. R. \& KaYe, N. B. 2001 Virtual origin correction for lazy turbulent plumes. J. Fluid Mech. 435, 377-396.

Hunt, J. C. R., Vrieling, A. J., Nieuwstadt, F. T. M. \& Fernando, H. J. S. 2003 The influence of the thermal diffusivity of the lower boundary on eddy motion in convection. J. Fluid Mech. 491, 183-205.

Kaminski, E., Tait, S. \& Carazzo, G. 2005 Turbulent entrainment in jets with arbitrary buoyancy. J. Fluid Mech. 526, 361-376.

Levine, J. 1959 Spherical vortex theory of bubble-like motion in cumulus clouds. J. Meteor. 16, 653-662.

Margolin, L. G., Rider, W. J. \& Grinstein, F. F. 2006 Modeling turbulent flow with implicit LES. J. Turbul. 7, 1-27.

Middleton, J. H. 1975 The asymptotic behaviour of a starting plume. J. Fluid Mech. 72, 753-771.

Morton, B. R., Taylor, G. I. \& Turner, J. S. 1956 Turbulent gravitational convection from maintained and instantaneous sources. Proc. R. Soc. Lond. A 234, 1-32.

Oran, E. S. \& Boris, J. P. 1993 Computing turbulent shear flows - a convenient conspiracy. Comp. Phys. 7, 523-533.

Porter, D. H., Pouquet, A. \& Woodward, P. R. 1992 Three-dimensional supersonic homogeneous turbulent: a numberical study. Phys. Rev. Lett. 68, 3156.

Ricou, F. P. \& Spalding D. B. 1961 Measurements of entrainment by axisymmetrical turbulent jets. J. Fluid Mech. 8, 21-32.

Scase, M. M., Caulfield, C. P. \& Dalziel, S. B. $2006 a$ Boussinesq plumes with decreasing source strengths in stratified environments. J. Fluid Mech. 563, 463-472.

Scase, M. M., Caulfield, C. P. \& Dalziel, S. B. 2008 Temporal variation of non-ideal plumes with sudden reductions in buoyancy flux. J. Fluid Mech. 600, 181-199.

Scase, M. M., Caulfield, C. P., Dalziel, S. B. \& Hunt, J. C. R. $2006 b$ Time-dependent plumes and jets with decreasing source strengths. J. Fluid Mech. 563, 443-461.

Scase, M. M., Caulfield, C. P., Dalziel, S. B. \& Hunt, J. C. R. $2006 c$ Plumes and jets with time-dependent sources in stratified and unstratified environments. In Proceedings of 6th International Symposium on Stratified Flows (ed. G. N. Ivey), University of Western Australia, Perth, Australia, pp. 112-117.

Scase, M. M., Caulfield, C. P., Linden, P. F. \& Dalziel, S. B. 2007 Local implications for self-similar turbulent plume models. J. Fluid Mech. 575, 257-265.

SCORER, R. S. 1954 The nature of convection as revealed by soaring birds and dragonflies. Q. J. R. Met. Soc. 80, 68-77.

Turner, J. S. 1957 Buoyant vortex rings. Proc. R. Soc. A 239, 61-75.

Turner, J. S. 1962 The 'starting plume' in neutral surroundings. J. Fluid Mech. 13, 356-368.

Turner, J. S. 1973 Buoyancy effects in fluids. Cambridge University Press.

Youngs, D. L. 1991 Three-dimensional numerical simulation of turbulent mixing by RayleighTaylor instability. Phys. Fluids A 4, 1312-1320.

Zeldovich, Y. B. 1937 The asymptotic laws of freely-ascending convective flows. Zhur. Eksper. Teor. Fiz. 7, 1463-1465 (in Russian). English translation In Selected Works of Yakov Borisovich Zeldovich, 1992 (ed. J. P. Ostriker), vol. 1, pp. 82-85. Princeton University Press. 